\title{
Some Approaches to Accounting for Incidental Carcass Discoveries in Non-Monitored Years using the Evidence of Absence Model
}

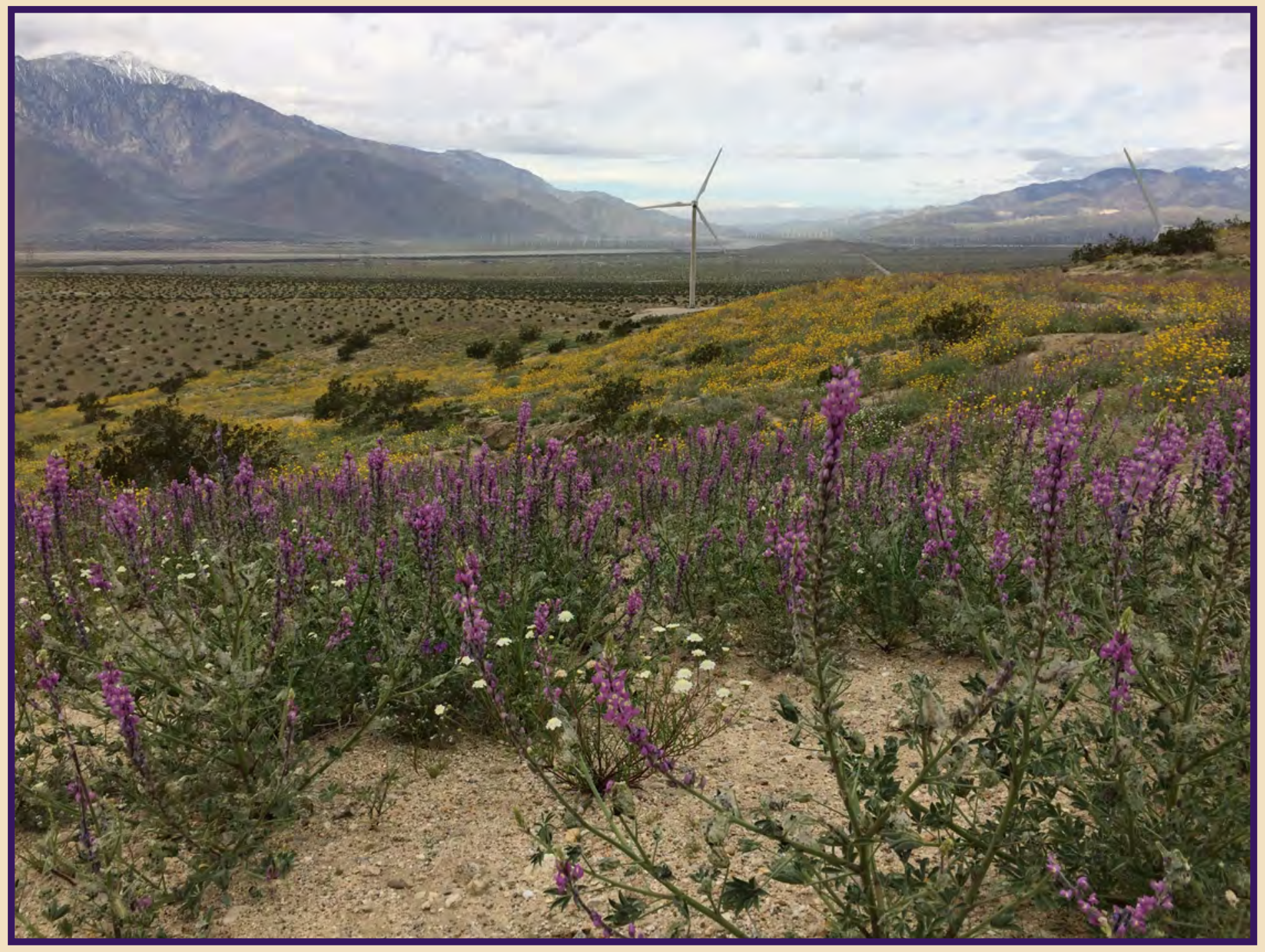

Open-File Report 2020-1027 
Cover: Wind turbines in Gorgonio Pass Wind Resource Area, California, March 20, 2019. (Photograph by Manuela Huso, U.S. Geological Survey.) 


\section{Some Approaches to Accounting for Incidental Carcass Discoveries in Non-Monitored Years using the Evidence of Absence Model}

by Daniel Dalthorp, Paul Rabie, Manuela Huso, and Andrew Tredennick

Open-File Report 2020-1027 


\section{U.S. Department of the Interior \\ DAVID BERNHARDT, Secretary}

\section{U.S. Geological Survey James F. Reilly II, Director}

U.S. Geological Survey, Reston, Virginia: 2020

For more information on the USGS-the Federal source for science about the Earth, its natural and living resources, natural hazards, and the environment-visit https://www.usgs.gov/ or call 1-888-ASK-USGS (1-888-275-8747).

For an overview of USGS information products, including maps, imagery, and publications, visit https://store.usgs.gov/.

Any use of trade, firm, or product names is for descriptive purposes only and does not imply endorsement by the U.S. Government.

Although this information product, for the most part, is in the public domain, it also may contain copyrighted materials as noted in the text. Permission to reproduce copyrighted items must be secured from the copyright owner.

Suggested citation:

Dalthorp, Daniel, Rabie, Paul, Huso, Manuela, and Tredennick, Andrew, 2020, Some approaches to accounting for incidental carcass discoveries in non-monitored years using the Evidence of Absence model: U.S. Geological Survey Open-File Report 2020-1027, 24 p., https://doi.org/10.3133/ofr20201027.

ISSN 2330-1258 (online) 


\section{Contents}

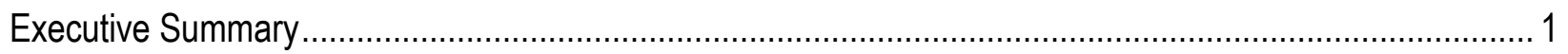

Three Methods to Account for Incidentals............................................................................ 1

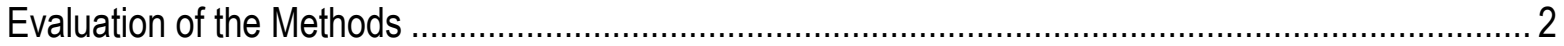

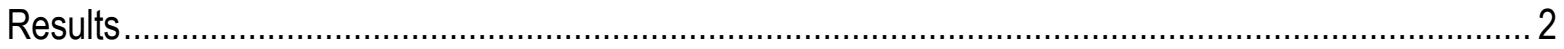

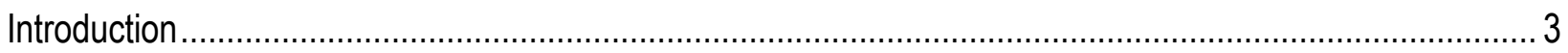

Three Approaches for Accounting for Incidental Carcasses ................................................................ 4

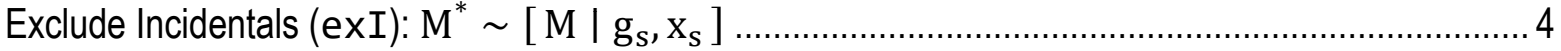

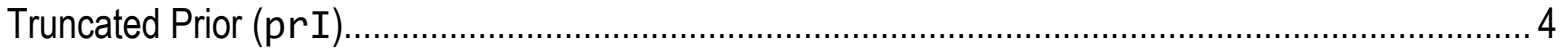

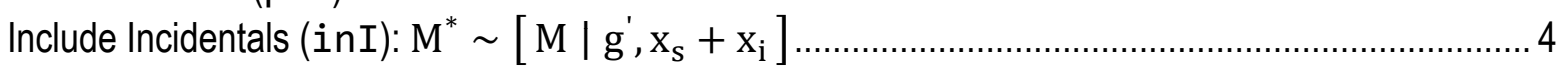

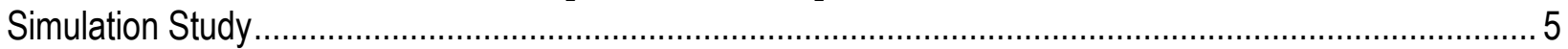

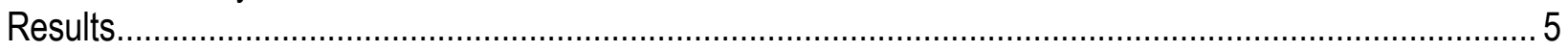

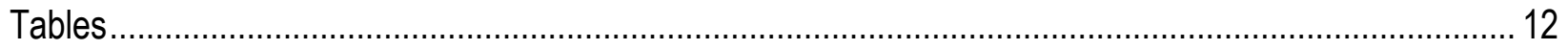

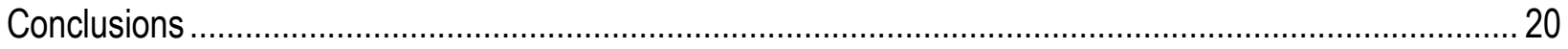

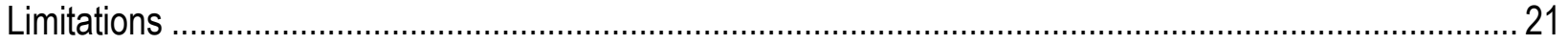

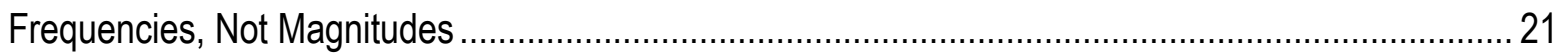

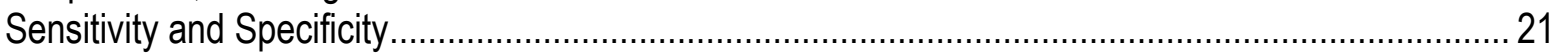

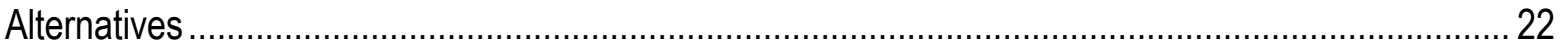

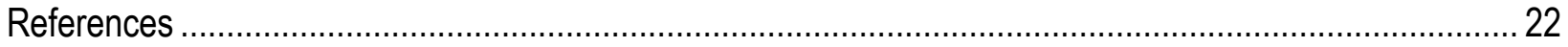

\section{Figures}

Figure 1. Convergence of estimator coverages when detection probability for incidental carcasses $\left(g_{i}\right)$

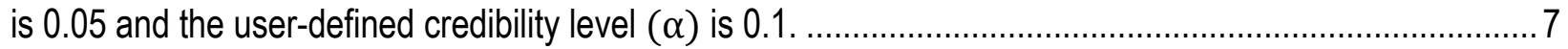

Figure 2. Estimator coverages for nominal 90 percent Cls. ......................................................... 8

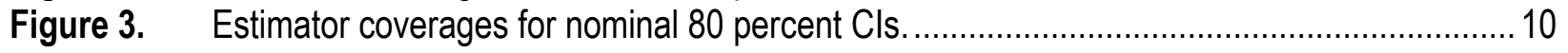

Figure 4. Estimator coverages for nominal 50 percent Cls. ......................................................... 11

\section{Tables}

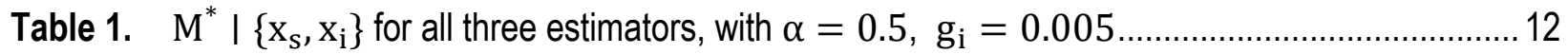

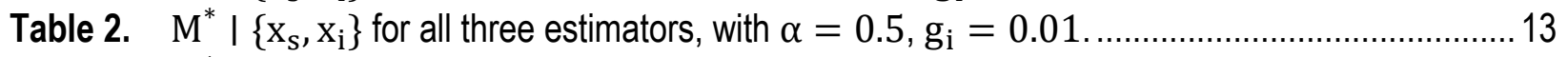

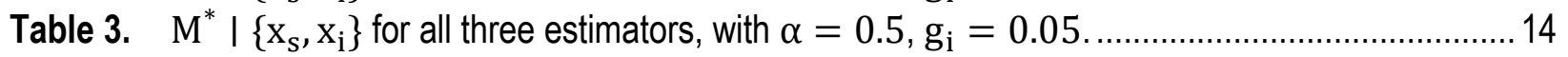

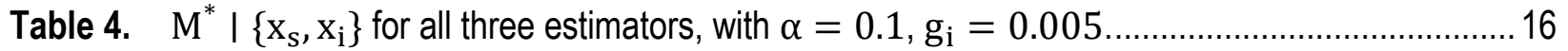

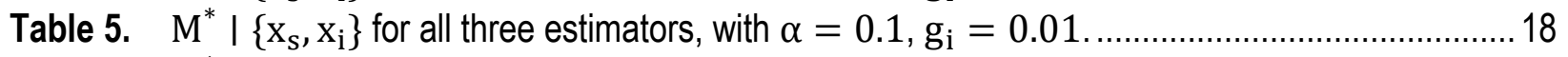

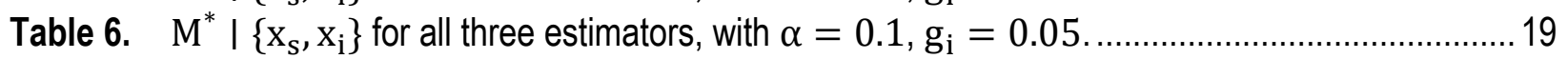

\section{Abbreviations}

$\begin{array}{ll}\text { EOA } & \text { evidence of absence } \\ \text { exI } & \text { Estimator } 1 \text { ("exclude incidentals") } \\ \text { inI } & \text { Estimator } 3 \text { ("include incidentals") } \\ \text { ITP } & \text { incidental take permit } \\ \text { PCM } & \text { post-construction monitoring } \\ \text { prI } & \text { Estimator } 2 \text { ("truncated prior") }\end{array}$




\section{Variables and Parameters}

$\alpha$

CI

$g_{s}$

$g_{i}$

$\lambda$

$\hat{\lambda}$

M

$M^{*}$

$x_{S}$

$x_{i}$ user-defined credibility level $(1-\alpha)$

credible interval (Bayesian analog of a confidence interval)

detection probability for formal PCM search process

detection probability for incidental carcasses

true annual mortality rate (for example, bats per year), Poisson distributed

estimated mortality rate

true number of fatalities

estimated minimum number of fatalities such that $\operatorname{Pr}\left(M \leq M^{*}\right) \geq 1-\alpha$

number of carcasses found during formal PCM searches

number of incidental carcasses found 


\title{
Some Approaches to Accounting for Incidental Carcass Discoveries in Non-Monitored Years using the Evidence of Absence Model
}

\author{
by Daniel Dalthorp ${ }^{1}$, Paul Rabie ${ }^{2}$, Manuela Huso ${ }^{1}$, and Andrew Tredennick²
}

\section{Executive Summary}

We evaluate three approaches to accounting for incidental carcasses when estimating an upper bound on total mortality $(M)$ as $M^{*}$ using the Evidence of Absence model (EoA; Dalthorp and others, 2017) to assess compliance with an Incidental Take Permit (ITP) (Dalthorp \& Huso, 2015) under a monitoring protocol that includes formal, dedicated carcass surveys that achieve an overall detection probability of $g_{s}=0.15$ in the first year, followed by 4 years with no formal monitoring but with carcasses potentially discovered incidentally by operations and maintenance crews in their normal course of activity or otherwise discovered outside the formal searches. We refer to carcasses discovered incidentally as "incidentals" and define $x_{i}$ as the count of incidentals. Similarly, we define $x_{s}$ as the number of carcasses found during the formal searches conducted the first year.

Three Methods to Account for Incidentals

The difficulty with incidentals is that they cannot simply be included in the carcass count that is used to calculate $M^{*}$ because the detection probability for incidentals is typically not known. This presents a statistical puzzle in that we know that the incidentals provide information about mortality rates, but it is difficult to quantify that information in the calculation of $M^{*}$. We explore three possibilities:

1. exI: exclude incidentals and calculate $M^{*}$ based solely on carcasses found in the formal search process (with detection probability $g_{s}$ );

2. prI: incorporate the information provided by incidentals into a truncated, informed prior distribution to be used in EoA;

3. inI: include incidentals in the carcass count and calculate $M^{*}$ based on all carcasses found $\left(x_{s}+x_{i}\right)$, whether in the formal search process (with detection probability $g_{s}$ or as incidentals (with detection probability $g_{i}$ ).

The first method (exI) is the default for EoA and is statistically valid. Carcasses discovered outside the monitored period or outside the search plots are accounted for by the spatial and temporal search coverage components in the EoA model. Although valid, the method loses statistical power as the number of unmonitored years accumulates.

\footnotetext{
${ }^{1}$ U.S. Geological Survey, Corvallis, Oregon

${ }^{2}$ Western Ecosystems Technology, Inc. (WEST), Cheyenne, Wyoming
} 
The second method (prI) makes use of some of the information in the incidentals without requiring an estimate or assumption about detection probability for incidental carcasses $\left(g_{i}\right)$. It is statistically valid and improves slightly on the statistical power of the first method. Also, because it does not require an estimate or assumption about $g_{i}$, it avoids the uncertainties involved in assuming a fixed value for $g_{i}$ and the expense of estimating $g_{i}$ if no fixed assumption is made about $g_{i}$.

The third method (inI) is an attempt to take maximal advantage of the information implicit in the incidental finds. To do so requires enhancing the information by either assuming a fixed value for $g_{i}$ or by adequately estimating it. If an assumed value for $g_{i}$ is badly mistaken (for example, assuming $g_{i}=0.05$ when it is really 0.005 or vice versa), there is substantial risk that $M^{*}$ will be grossly inaccurate. However, if it is known that $g_{i}$ is small (for example, $g_{i}<$ 0.01 ), and $g_{i}$ is assumed to be small, then misspecification of $g_{i}$ has little effect on the accuracy of inI coverage probabilities. Because information in the incidentals is more fully incorporated into estimates, discovery of incidentals in non-monitored years can have a strong impact on $M^{*}$, providing greater opportunity to detect high fatality rates that went unnoticed in the first year of monitoring but increasing the probability of an $M^{*}$ that is much higher than the true $M$.

\section{Evaluation of the Methods}

EoA was designed to calculate the upper bounds $\left(M^{*}\right)$ of $100(1-\alpha) \%$ credibility intervals for mortality $(M)$, which can be interpreted as the maximum number of fatalities that is compatible with the data. A total mortality that exceeds $M_{\alpha}^{*}$ can be effectively ruled out with $100(1-\alpha) \%$ credibility. The natural test for EoA-type models is on the accuracy of $M_{\alpha}^{*}$, that is, whether the probability that $M$ exceeds $M^{*}$ is close to $\alpha$. We call the true probability that $M_{\alpha}^{*} \geq$ $M$ the "coverage probability" or simply "coverage" and call $1-\alpha$ the nominal or target coverage. We conducted simulations to test the coverage probabilities for the three methods under a wide variety of scenarios under a general framework of:

- formal PCM searches conducted in the first year, with detection probability of $g_{s}=0.15$;

- 4 years without formal monitoring

$\circ$ actual detection probabilities for incidentals ranging from $g_{i}=0.001$ to 0.05

$\circ$ assumed detection probabilities ( $\mathrm{i} n \mathrm{I}$ only) ranging from 0.001 to 0.05

- actual mortality rates ranging from $\lambda=0.5 /$ year to $10 /$ year;

- $\alpha$ values of $0.1,0.2$, and 0.5 .

\section{Results}

We found that coverages for all three estimators (assuming $g_{i}$ known in inI) converged to the target $1-\alpha$ as $\lambda$ increased, with rate of convergence dependent on $\alpha$. Estimators exI and prI had similar coverage in almost all cases, with prI outperforming exI in most of the few cases where they differed. Both estimators over-covered (implying a tendency for $M^{*}$ to be too large) when the annual take $(\lambda)$ was small and credibility level $(1-\alpha)$ was large. As $\lambda$ increased, both estimators converged on nominal coverage at close to the same rate when $\alpha$ was 0.5 .

When $g_{i}$ was known, estimator inI had more accurate coverage probabilities than did exI and prI in most scenarios. In particular, it had much less of a tendency to overestimate when $\lambda$ was small and $g_{i}$ was large. This is because inI directly and efficiently incorporates the information from the incidentals into the estimates. However, its advantage appeared to largely 
evaporate when desired credibility was 50 percent, in which case inI represented only a marginal improvement over exI or prI.

The relatively good performance of estimator inI in many of the simulation scenarios depends on $g_{i}$ being known, which is usually an unrealistic assumption. When $g_{i}$ is misspecified or poorly estimated, inI can be strongly biased. Because the process of detecting incidentals is difficult to predict, designing and conducting trials to accurately estimate $g_{i}$ would require careful thought. In theory, $g_{i}$ could be estimated using simplified binomial search trials that do not require follow-up searches to monitor the fates of carcasses after initial placement. This would involve placement of trial carcasses throughout the monitoring period and using the fraction of those carcasses that were discovered incidentally as an estimate of $g_{i}$ (after accounting for search coverage).

\section{Introduction}

Wind energy facility operators typically conduct post-construction fatality monitoring (PCM) studies at wind energy facilities to evaluate the direct impacts to birds and bats, which may collide with wind turbines. Counts of carcasses are analyzed with data from experimental trials designed to evaluate detection bias (imperfect searcher efficiency, carcass removal, unsearched areas, etc.) to arrive at an estimate of total mortality and mortality rate at a site. When the goal is to provide assurance of compliance with an incidental take permit of a rare species (for which carcass counts may be low or even zero), the Evidence of Absence (EoA; Dalthorp and others, 2017; Dalthorp and Huso, 2015) estimator is the most appropriate statistical estimator available. In this context, EoA is not designed to give a point estimate of how many rare individuals were killed, but to calculate $M^{*}$ and credible intervals, which are the Bayesian analog to the confidence intervals of classical statistics. In practice, $M^{*}$ is often used to determine whether projects are in compliance with their incidental take permits (ITPs) and to determine the mitigation debits accruing to a permit holder. In mathematical terms, $M_{\alpha}^{*}$ is defined as the minimum number of fatalities such that $\operatorname{Pr}\left(M \leq M^{*} \mid X=x ; g=\widehat{g}\right) \geq 1-\alpha$ for the user's choice of credibility level, $1-\alpha$ where $M$ is the true mortality of the rare species, $x$ is the number of carcasses found in a search process for which the detection probability has been estimated as $\widehat{g}$ (Dalthorp and others, 2017). In lay terms, EoA is saying you have a $1-\alpha$ chance that true mortality was $\leq M^{*}$ given your search process and what was discovered. We refer to this value as $M^{*}$ throughout, including subscripts as needed to differentiate among estimators or to distinguish credibility levels.

Finding incidental carcasses (defined as bird or bat carcasses found outside of prescribed PCM searches) at a wind energy facility has been the source of discussion regarding how they should be treated in the context of EoA. Observations of incidental carcasses are not data in the sense that they were not observed as part of the data collection process that is modeled in EoA. Indeed, the EoA framework is designed to account, through $\widehat{g}$, for carcasses that likely exist but were not found during PCM searches, which would include incidental carcasses. However, when the purpose of monitoring is to evaluate compliance with the Endangered Species Act and associated ITP and to determine mitigation debits for permit holders, these incidental finds can represent valuable information.

The question we address here is: How can incidental carcasses be treated in the context of EoA while maintaining the strong statistical properties of the estimator? In this report, we focus exclusively on one specific monitoring plan in which PCM monitoring is conducted in the first year with an overall detection probability of $g_{s}=0.15$, followed by 4 years in which no formal 
PCM is conducted, but the potential exists for bats to be discovered incidentally by workers at the site. We describe three approaches to accounting for incidental carcasses when estimating $M^{*}$ in the EoA framework to assess compliance with an ITP (Dalthorp and Huso, 2015). We describe a probability-based simulation we conducted to assess the three approaches for handling incidentals in terms of coverage probabilities; that is, the accuracy of the level of credibility given in statements that can be made using EoA like "Having searched for Indiana bats with an overall detection probability of $g$ and having found none, we can assert with 50 percent credibility that no more than five were taken in this period." We provide results and discussion of results and conclude with recommendations on approaches to interpreting incidental finds in a management context where the objective is to be reasonably sure-with $100(1-\alpha) \%$ credibility - that permitted take is not being exceeded.

\section{Three Approaches for Accounting for Incidental Carcasses}

\section{Exclude Incidentals (exI): $M^{*} \sim\left[M \mid g_{s}, x_{s}\right]$}

This is the standard EoA calculation of $M^{*}$. The modeling framework of EoA already accounts for carcasses that are missed due to unsearched areas, unsearched periods of time, carcass removal, or imperfect searcher efficiency, and that the exI approach ("exclude incidentals") is well-supported by statistical theory. An incidental carcass represents one of those missed and can be thought of as accounted for in the estimator. EoA acknowledges that some carcasses may be missed in the searches, and an incidental represents serendipitous discovery of one that was missed.

\section{Truncated Prior (prI)}

The prI estimator makes use of the information that total mortality must be greater than or equal to the number of incidentals found by truncating the lower bound of EoA's prior distribution to exclude the possibility that $M<x_{i}$. Instead of using an integrated binomial reference prior $(\operatorname{Pr}(M=m) \propto \sqrt{m+1}-\sqrt{m})$ that EoA uses as a default, prI uses a prior that has probability 0 for $M<x_{i}$ and $\operatorname{Pr}(M=m) \propto 1 / \sqrt{m}$ (a Jeffreys prior) for $m \geq x_{i}$. When the number of incidentals $\left(x_{i}\right)$ does not exceed the number of carcasses found in formal searches $\left(x_{s}\right)$, the prI estimator is virtually indistinguishable from the traditional exI. The differences between exI and prI are significant only when $x_{i}>x_{s}$ and $x_{s}$ is small. This situation would be expected to be rare except when the detection probability for incidentals is not small in relation to $g_{s}$.

Include Incidentals (inI): $M^{*} \sim\left[M \mid g^{\prime}, x_{s}+x_{i}\right]$

Including incidentals in the carcass survey count requires accounting for the detection probability of the incidentals, which we define as carcasses that are discovered outside of standardized search plots or in non-monitored years. In either case, $g_{s}$ in the model should be slightly adjusted and replaced with a $g^{\prime}$ that includes a component that reflects detection probability of incidentals. If the detection probability for survey carcasses $\left(g_{s}\right)$ is substantially higher than detection probability for incidentals $\left(g_{i}\right)$, this approach would be fairly robust to misspecification of $g_{i}$, and $g_{i}$ could be assumed to be some fixed value. However, as the nonmonitored years accumulate, the overall proportion of carcasses found in the carcass surveys 
decreases ( $g_{s}$ in year $1, g_{s} / 2$ for carcasses through the first two years, etc.), and the sensitivity to misspecification of $g_{i}$ increases. Our simulations include evaluation of effect of misspecification of $g_{i}$.

\section{Simulation Study}

We assess (via simulation) how the estimators perform under various realistic scenarios that are rooted in probability and evaluate their performance in terms of coverage. Particularly, we adopt the basic framework of annual mortalities as $M \sim \operatorname{Poisson}(\lambda), 1$ year of PCM monitoring at $g_{s}=0.15$ followed by $1-4$ non-monitored years, during which the probability of finding incidentals is $>0$. Scenarios include all combinations of $\lambda \in\{0.5,1,1.5, \ldots, 10\}$ and detection probabilities for incidental carcasses $g_{i} \in\{0.001,0.0025,0.005,0.01,0.025,0.05\}$. In addition, estimator inI is based on an assumed $g_{i}$ taken from the same set as the actual $g_{i}$. Thus, for example, in some scenarios inI assumed $g_{i}$ is correct and in others $g_{i}$ is mis-specified. The other estimators make no assumptions about $g_{i}$. In the first year, when the carcass surveys are conducted, the detection probability for the carcass surveys is assumed to be $g_{s}=0.15$, and carcasses that are not found in the searches are available for detection as incidentals. The probability of incidental detections in the first year is likely to be lower than in later years because most of the would-be incidental discoveries are made by the search teams instead. We assume that the detection for incidentals in the first year is $g_{i} / 3$. The denominator is arbitrary, but the estimator is only weakly dependent on that assumption unless $g_{i}$ is not small in relation to $g_{s}$. For inI, we then take the assumed $g$ to be $g^{\prime}=g_{s}+\left(1-g_{s}\right) g_{i} / 3$ for the first year and $\left(g_{s}+\left(1-g_{s}\right) g_{i} / 3+(y-1) g_{i}\right) / y$ for cumulative totals over $y$ years.

We evaluate the estimators in terms of coverage probabilities of $M_{\alpha}^{*}$ for $M$ with $\alpha \in$ $\{0.1,0.2,0.5\}$, which reflects the intent and common implementation of EoA as a tool for determining compliance. In addition, extensive tables show $M^{*}$ values that may be useful in evaluating mitigation obligations. This general framework, which includes models for discovery of incidentals, allows for evaluation of the estimators rooted in probability theory.

\section{Results}

Under most of the conditions that we tested, the estimators performed similarly. The similarities began to break down as the detection probability for incidentals $\left(g_{i}\right)$ increased to 0.05 , and the distinctions were most pronounced when $\alpha=0.1$. Figure 1 shows coverage probabilities for the three estimators when $g_{i}=0.05, y=5$, and $\alpha=0.1$ for $\lambda$ from 0.5 to $\lambda=$ 60 per year, a mortality rate that is unlikely to be seen in practice but is useful for seeing convergence of the estimators to nominal coverage.

A perfect estimator would cover $M$ exactly $100(1-\alpha) \%$ of the time, but exact coverage is rarely possible for discrete distributions, especially when counts are small. Estimator overcoverage (above the blue target) means that $M^{*}$ exceeds $M$ more frequently than expected, which means the estimator tends to overestimate for that scenario. Similarly, coverage below the target indicates a tendency to underestimate. Lines represent coverage probabilities of $M_{\alpha}^{*}$ for cumulative mortality through $y$ years. The degree of overestimation or underestimation in particular cases can be seen in tables $1-6$.

Additionally, a $g_{i}$ of 0.05 is probably an upper bound on what might occur for bats, and $\alpha=0.1$ is more stringent than the more commonly used $\alpha=0.5$ or 0.2 . Thus, figure 1 represents the scenarios with the most extreme divergence among the estimators and serves to highlight the 
qualitative differences in how the estimators make use of the information in the incidentals. Quantitative differences among the estimators depend on specific combinations of $g_{i}$ and $\alpha$, as illustrated in figures $2-4$.

In all cases, coverage probabilities for exI and prI converged to the target coverage as $\lambda$ increased. Although virtually identical over most of the range of $\lambda$, exI and prI diverge from each other in figure 1 when $\lambda$ was between, roughly, 8 and 15 . Under these conditions, prI gave somewhat higher coverages than exI, reflecting a tendency to give higher estimates. This gives the prI model slightly greater power than exI to detect large $\lambda$ 's in the non-monitored years.

When mortality rates are not very high $(\lambda<7$ in the figure $)$ - which is likely in practice - estimators exI and prI have a strong tendency to overestimate the total mortality over 5 years, with coverage probability of 100 percent when $g_{i}=0.05$ and $\alpha=0.1$. The problem is that the information content in the formal carcass surveys in year 1 is progressively diluted as the non-monitored years accrue, and the overall detection probability for carcasses arriving over the course of 5 years decreases to 3 percent. After accounting for the uncertainty inherent in such a small $g$, it becomes difficult to rule out mortality of $M>\lambda \cdot 5$ when $\lambda$ is not large.

The inI estimator can remedy some of the tendency for exI and prI to overestimate under the conditions in figure 1 . With $g_{i}=0.05$ the information content in the incidentals is potentially high, but harvesting that information efficiently requires knowing or reliably estimating $g_{i}$. If $g_{i}$ is known, inI converges to nominal coverage more rapidly than the other two estimators and has substantially less of a tendency toward over-estimation. However, this requires that detection probability for incidentals is known or reliably estimated.

In figures 2-4, coverage probabilities for $M_{\alpha}^{*}$ are shown for the three estimators for $0.5 \leq$ $\lambda \leq 10$ and $g_{i} \in\{0.001,0.0025,0.005,0.01,0.02,0.05\}$. The black and green lines represent coverages for exI and prI, resp. The solid red lines represent coverages for inI when the value assumed for $g_{i}$ in inI is correct; broken red lines are for inI when $g_{i}$ is misspecified. The topmost red line is for an assumed $g_{i}$ of 0.001 , and successively lower lines are for $g_{i}=0.0025$, $0.005,0.01,0.02,0.05$, respectively. The horizontal, light blue line represents the nominal, target coverage $(1-\alpha)$. In many panels, the coverages for one or more of the estimators are nearly identical for most, if not all, of the values of $\lambda$. As a result, sometimes one or more lines appear to be missing. This is because they are entirely coincident with a line that was drawn later. The lines were drawn in order: exI first (black), inI next (red), and prI last (green). 


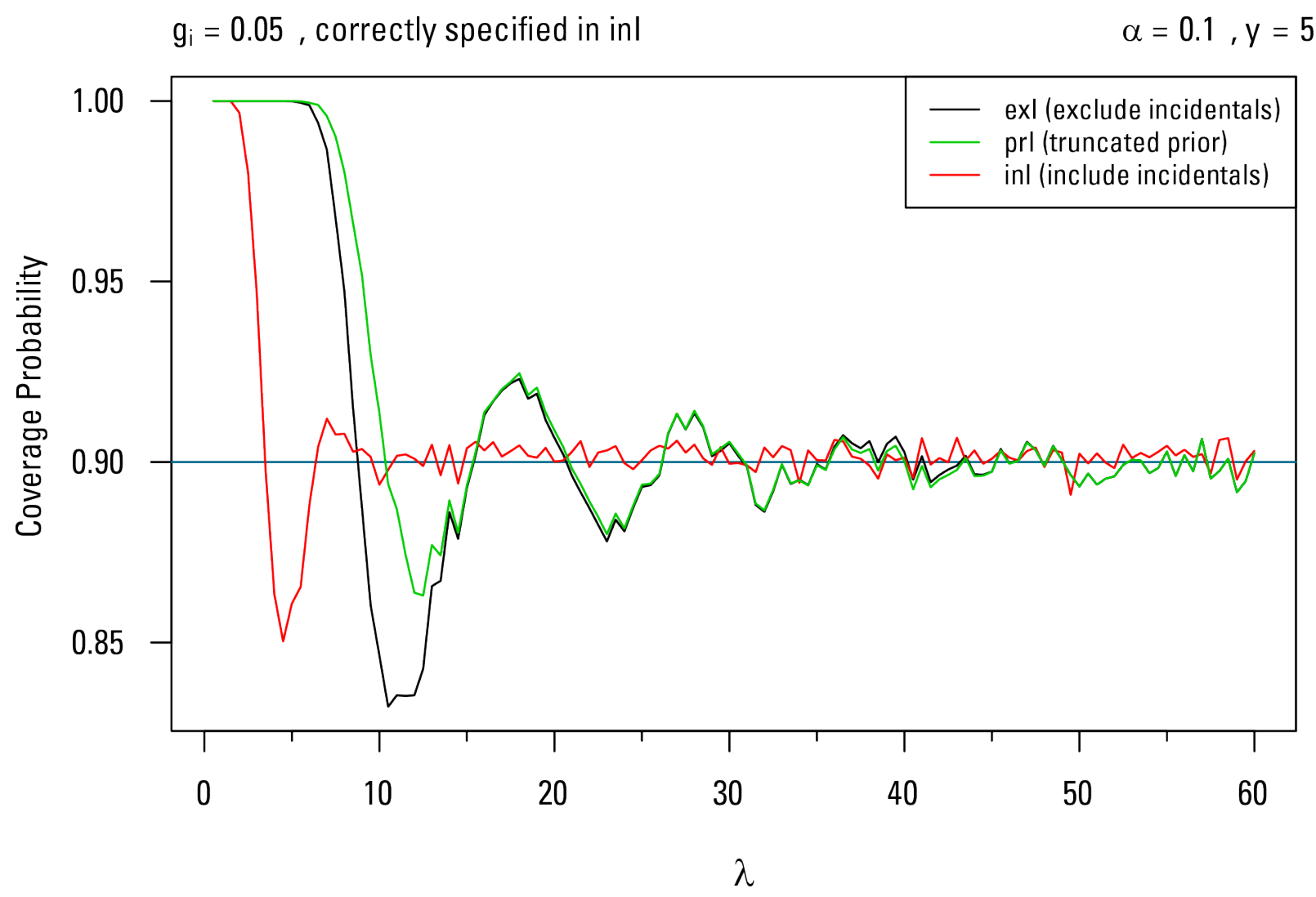

Figure 1. Convergence of estimator coverages when detection probability for incidental carcasses $\left(g_{i}\right)$ is 0.05 and the user-defined credibility level $(\alpha)$ is 0.1 . [Horizontal blue line indicates target coverage.]

In all cases, estimator exI is independent of the detection probability for incidentals $\left(g_{i}\right)$, so the black lines are identical within each column in the panels. When $g_{i}$ is small (for example, $g_{i} \leq 0.01$ ), discovery of incidentals is not likely, so the prior in prI rarely needs adjustment. As a result, in the small $g_{i}$ scenarios, prI and exI are virtually indistinguishable. This is reflected in the figures by a virtual absence of a black line for exI, which becomes hidden by the green line for prI. Additionally, whenever $x_{i} \leq x_{s}$ the adjustment to the prior in prI has trivial impact on $M^{*}$ because the posterior distribution for $M$ will automatically assign probability 0 to values of $M$ that are $<x_{s}$, so the incidentals do not provide information that affects the estimation. In other words, truncating the prior when the posterior would perform the same (or a possibly more pronounced) truncation has virtually no effect.

Coverages (nominal 90 percent) for exI and prI are largely indistinguishable (fig. 2) in the simulation scenarios except when $g_{i}$ is relatively large, in which case prI had a greater tendency to overestimate than did exI. When $\lambda>9$, exI had a slight tendency to underestimate.

In these scenarios where $g_{i}$ is correctly specified for in I (solid red lines), convergence to nominal coverage is faster than it is for the other two estimators, especially when $g_{i}$ is larger (bottom rows of figures). This means that when $\alpha=0.1$ and $\lambda$ is small, inI shows much less of a tendency to overestimate than do exI and prI. This is because, by assigning a detection probability to incidentals and incorporating them into the estimator, inI is able to attain a larger $g$ value than either exI or prI, thereby tending to give smaller $M^{*}$ values, especially when $g_{i}$ and $\lambda$ are large, in which case the incidentals carry more information, which inI is able to leverage into greater accuracy (fig. 2). 
$\longrightarrow \operatorname{exl} \longrightarrow \mathrm{prl} \longrightarrow$ inl

$\alpha=0.1$

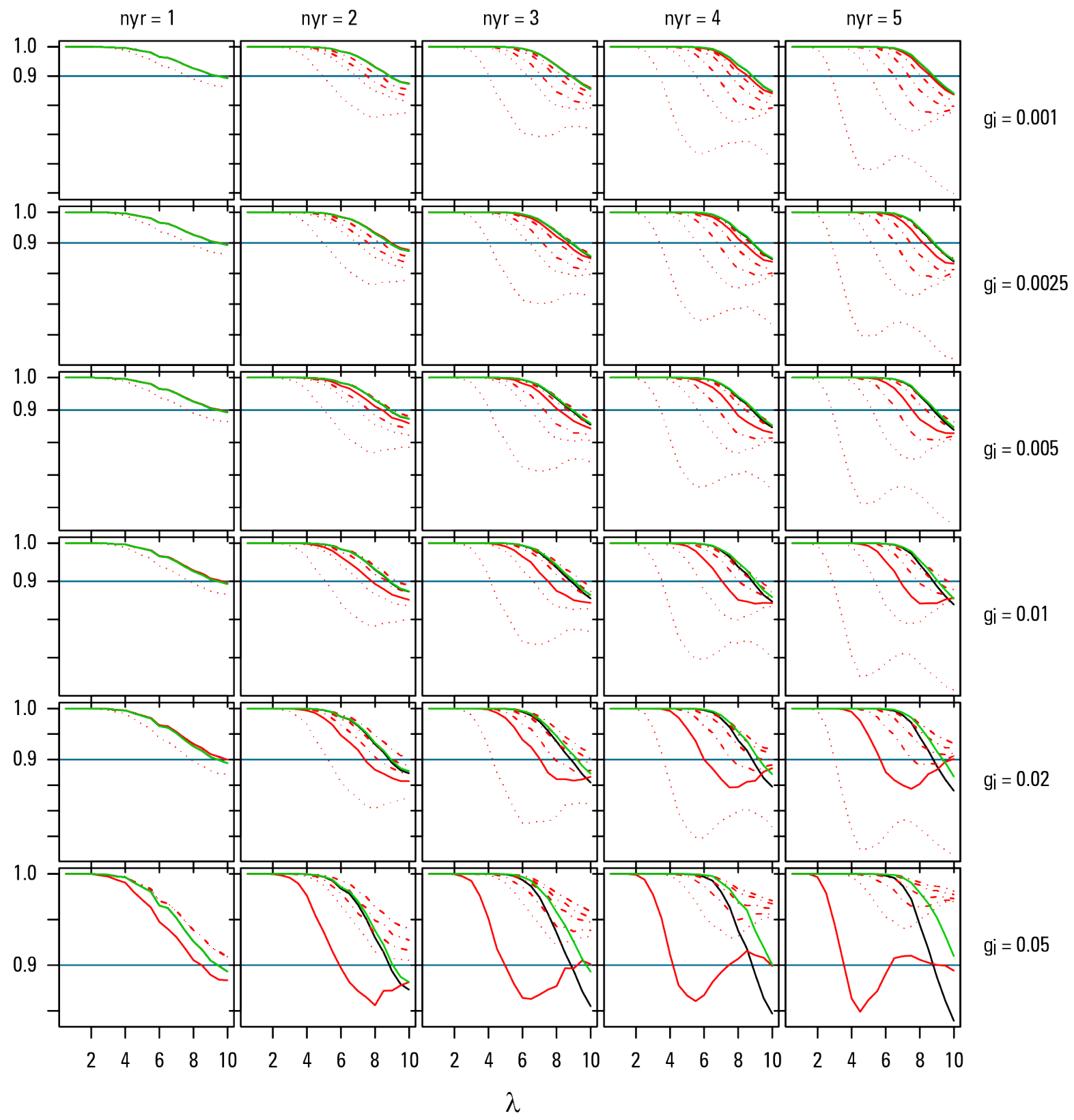

Figure 2. Estimator coverages for nominal 90 percent Cls. [Red lines are for Estimator 3 (inI) when detection probability for incidental carcasses $\left(g_{i}\right)$ is specified as $0.001,0.0025,0.005,0.01,0.02$, or 0.05 (top to bottom lines). Solid lines are for $g_{i}$ correctly specified and broken lines for detection probability for incidental carcasses $g_{i}$ misspecified (dashed for assumed $g_{i} \leq 0.01$ and dotted for $g_{i} \geq 0.02$ ). Blue lines indicate the target coverage.] 
When $g_{i}$ is misspecified, the performance of inI is notably less impressive than when $g_{i}$ is correctly assumed. For example, when true $g_{i}$ is 0.01 (third row of panels in fig. 2) and $g_{i}$ is assumed to be $>0.01$ (dotted red lines below the solid line), inI has a fairly strong tendency to underestimate, and when true $g_{i}=0.01$ and $g_{i}$ is wrongly assumed to be 0.001 or 0.0025 , the performance advantage of inI over exI and prI vanishes, as reflected in the dashed red lines in the fourth row of panels (fig. 2).

In general, with $\alpha=0.1$, if true $g_{i}$ is small ( $g_{i} \leq 0.01$, top four rows of panels) and $g_{i}$ is assumed to be small (dashed or solid red lines), then inI performs similarly to exI and prI but tends to have lower estimates where they differ.

When true $g_{i}=0.05$ but is assumed to be small, inI tends to overestimate, matching or even exceeding the tendency of prI to overestimate when $\lambda$ is very large $(\lambda>7$ or 8$)$, and $g_{i}$ is assumed to be very small $\left(g_{i} \leq 0.005\right)$.

Qualitatively, the same kinds of patterns repeat when $\alpha=0.2$ or $\alpha=0.5$, but the nuances differ. 
$\longrightarrow$ exl $\longrightarrow$ prl $\longrightarrow$ inl

$\alpha=0.2$

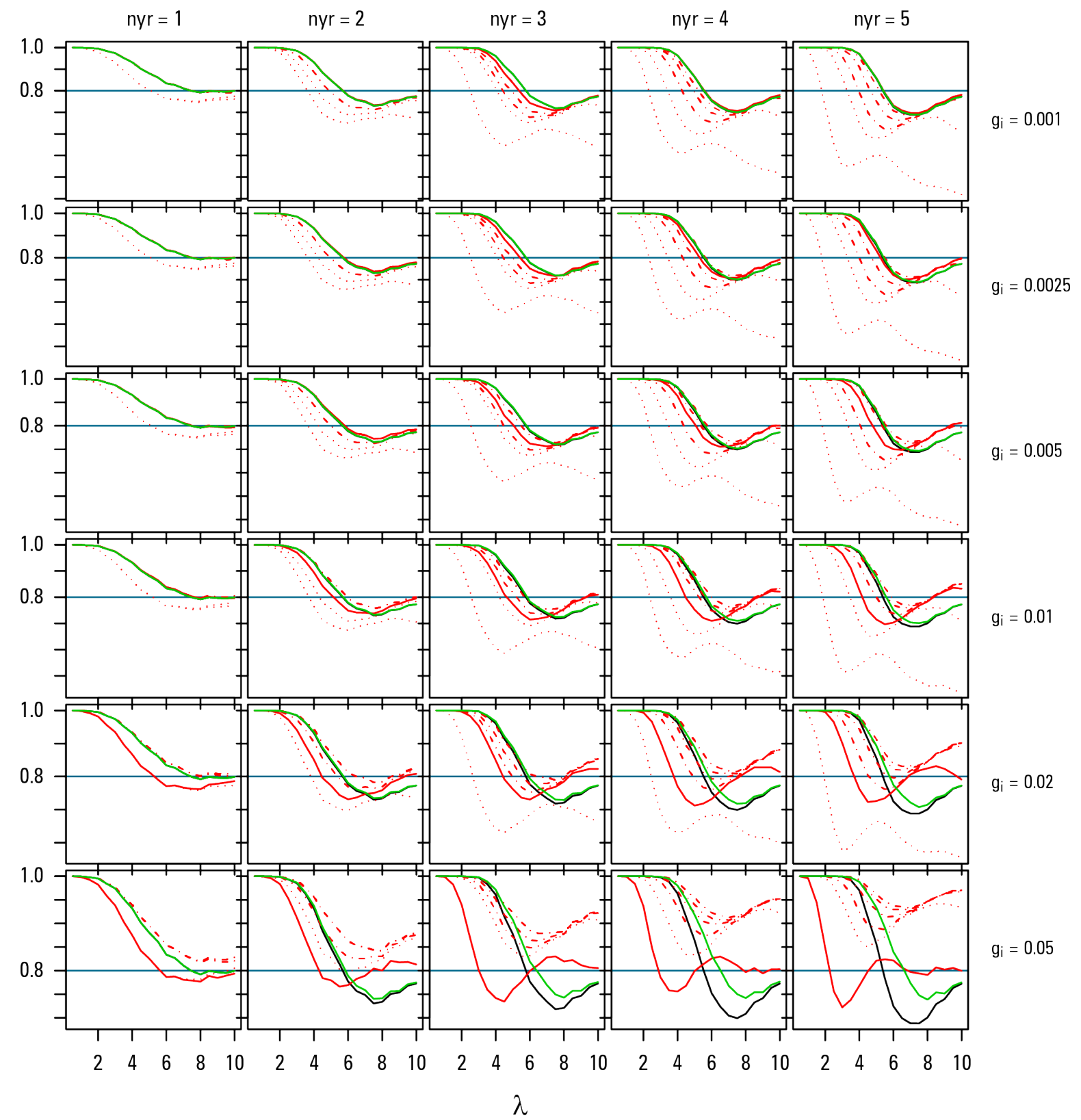

Figure 3. Estimator coverages for nominal 80 percent Cls. [Red lines are for in I when the detection probability for incidental carcasses $\left(g_{i}\right)$ is specified as $0.001,0.0025,0.005,0.01,0.02$, or 0.05 (top to bottom lines). Solid lines are for $g_{i}$ correctly specified and broken lines for $g_{i}$ misspecified (dashed for assumed $g_{i} \leq 0.01$ and dotted for $g_{i} \geq 0.02$ ). Blue lines indicate the target coverage.] 


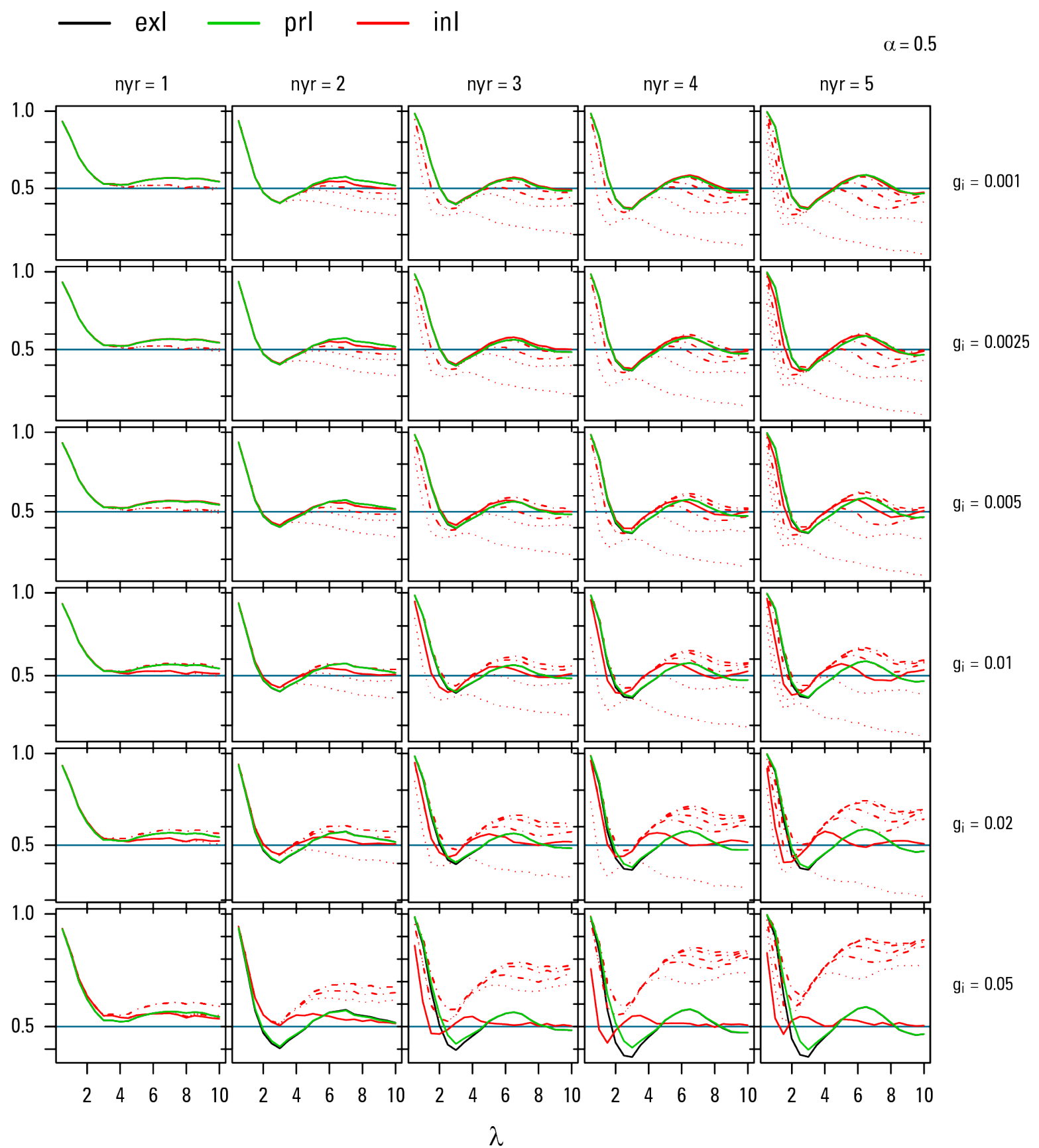

Figure 4. Estimator coverages for nominal 50 percent Cls. [Red lines are for in I when incidental carcasses $\left(g_{i}\right)$ is specified as $0.001,0.0025,0.005,0.01,0.02$, or 0.05 (top to bottom lines). Solid lines are for $g_{i}$ correctly specified, and broken lines for $g_{i}$ misspecified (dashed for assumed $g_{i} \leq 0.01$ and dotted for $g_{i} \geq 0.02$ ). Blue lines indicate the target coverage.]

When $\alpha=0.5$ (fig. 4), overall performances of exI and prI are virtually indistinguishable. When $g_{i}$ is correctly specified, inI outperforms both exI and prI, but the differences are slight except when $g_{i}=0.05$ and $\lambda$ is small.

As when $\alpha=0.1$ or 0.2 , misspecification of $g_{i}$ degrades the performance of estimator inI. If true $g_{i} \leq 0.01$ and $g_{i}$ is likewise assumed to be small, the overall performance of inI is 
comparable to that of exI or prI. However, when $g_{i}$ is not small $(\geq 0.02)$ but is assumed to be small (dashed lines in bottom two rows of panels in fig. 4), inI has a strong tendency to overestimate, and when true $g_{i} \leq 0.01$ but is assumed to be not small ( $g_{i} \geq 0.02$, dotted red lines in top four rows of panels in fig. 4), inI has a strong tendency to underestimate.

\section{Tables}

Mortality estimates for all three estimators $\left(M_{\mathrm{exI}}^{*}, M_{\mathrm{exI}}^{*}\right.$, and $\left.M_{\mathrm{inI}}^{*}\right)$ as a function of specific numbers of carcasses found in regular PCM searches $\left(x_{s}\right)$, incidental carcass discoveries $\left(x_{i}\right)$, detection probabilities $\left(g_{i}\right)$, and credibility levels $(1-\alpha)$ are listed in the following series of tables. Also included in the tables are the probabilities that each given combination of $x_{s}$ and $x_{i}$ occur, depending on the actual annual fatality rate $(\lambda=2,5$, or 10$)$ and number of monitored years ( 1 or 5). Tables with all combinations of $g_{i} \in\{0.005,0.01,0.05\}$ and $\alpha \in\{0.5,0.1\}$ are presented below.

The tables are dense with information, and little interpretation is provided. Instead, they are provided to allow readers to investigate probabilities and magnitudes of potential estimation errors. A discussion of Table 1 provides guidance on interpretation.

Table 1 shows the $M_{0.5}^{*}$ values for all three estimators when $g_{i}=0.005$ as a function of $x_{s}$ and $x_{i}$ and the number of years of data. It is assumed that $g_{i}$ is correctly specified in inI. The probabilities of each combination of $x_{s}$ and $x_{i}$ are shown for each $\lambda \in\{2,5,10\}$.

Table 1. $M^{*} \mid\left\{x_{s}, x_{i}\right\}$ for all three estimators, with $\alpha=0.5, g_{i}=0.005$.

\begin{tabular}{|c|c|c|c|c|c|c|c|c|c|c|c|c|c|}
\hline \multirow[b]{3}{*}{$x_{s}$} & \multirow[b]{3}{*}{$x_{i}$} & \multirow[b]{3}{*}{$\boldsymbol{M}_{\mathrm{exI}}^{*}$} & \multirow[b]{3}{*}{$\boldsymbol{M}_{\mathrm{prI}}^{*}$} & \multirow[b]{3}{*}{$\boldsymbol{M}_{\mathrm{inI}}^{*}$} & \multicolumn{3}{|l|}{1 year } & \multicolumn{6}{|c|}{5 years } \\
\hline & & & & & \multicolumn{3}{|c|}{$\operatorname{Pr}\left(x_{s}, x_{i} \mid \lambda, g_{i}=0.005\right)$} & \multirow[b]{2}{*}{$\boldsymbol{M}_{\mathrm{exI}}^{*}$} & \multirow[b]{2}{*}{$\boldsymbol{M}_{\mathrm{prI}}^{*}$} & \multirow[b]{2}{*}{$\boldsymbol{M}_{\mathrm{inI}}^{*}$} & \multicolumn{3}{|c|}{$\operatorname{Pr}\left(x_{s}, x_{i} \mid \lambda, g_{i}=0.005\right)$} \\
\hline & & & & & $\lambda=2$ & $\lambda=\mathbf{5}$ & $\lambda=10$ & & & & $\lambda=2$ & $\lambda=\mathbf{5}$ & $\lambda=\mathbf{1 0}$ \\
\hline 0 & 0 & 1 & 1 & 1 & 0.7405 & 0.4687 & 0.2195 & 7 & 7 & 6 & 0.7110 & 0.4234 & 0.1798 \\
\hline 1 & 0 & 8 & 8 & 8 & 0.2208 & 0.3541 & 0.3296 & 39 & 39 & 34 & 0.2123 & 3210 & 2689 \\
\hline 2 & 0 & 14 & 14 & 14 & 0.0325 & 0.1300 & 0.2483 & 72 & 72 & 63 & 0.0311 & 0.1183 & 0.2043 \\
\hline 3 & 0 & 21 & 21 & 21 & 0.0032 & 0.0330 & 0.1249 & 105 & 105 & 92 & 0.0030 & .0297 & 0.1024 \\
\hline 4 & 0 & 27 & 27 & 27 & 0.0002 & 0.0061 & 0.0457 & 139 & 139 & 121 & 0.0002 & 0.0056 & 0.0373 \\
\hline 5 & 0 & 34 & 34 & 34 & 0.0000 & 0.0009 & 0.0143 & 172 & 172 & 151 & 0.0000 & 0.0008 & .0119 \\
\hline 6 & 0 & 41 & 41 & 40 & 0.0000 & 0.0001 & 0.0035 & 205 & 205 & 180 & 0.0000 & .0001 & .0028 \\
\hline 7 & 0 & 47 & 47 & 47 & 0.0000 & 0.0000 & 0.0007 & 239 & 239 & 209 & 0.0000 & 0.0000 & 0.0006 \\
\hline 8 & 0 & 54 & 54 & 54 & 0.0000 & 0.0000 & 0.0001 & 272 & 272 & 238 & 0.0000 & 0.0000 & 0.0001 \\
\hline 0 & 1 & 1 & 3 & 8 & 0.0022 & 0.0036 & 0.0029 & 7 & 10 & 34 & 0.0309 & 0.0466 & 0.0382 \\
\hline 1 & 1 & 8 & 7 & 14 & 0.0006 & 0.0023 & 0.0047 & 39 & 39 & 63 & 0.0088 & 0.0337 & 0.0586 \\
\hline 2 & 1 & 14 & 14 & 21 & 0.0001 & 0.0009 & 0.0032 & 72 & 72 & 92 & 0.0014 & 0.0119 & 0.0426 \\
\hline 3 & 1 & 21 & 21 & 27 & 0.0000 & 0.0002 & 0.0017 & 105 & 105 & 121 & 0.0002 & 0.0034 & 0.0218 \\
\hline 4 & 1 & 27 & 27 & 34 & 0.0000 & 0.0000 & 0.0007 & 139 & 139 & 151 & 0.0000 & 0.0006 & 0.0082 \\
\hline 5 & 1 & 34 & 34 & 40 & 0.0000 & 0.0000 & 0.0002 & 172 & 172 & 180 & 0.0000 & 0.0001 & 0.0024 \\
\hline 6 & 1 & 41 & 41 & 47 & 0.0000 & 0.0000 & 0.0000 & 205 & 205 & 209 & 0.0000 & 0.0000 & 0.0006 \\
\hline 7 & 1 & 47 & 47 & 54 & 0.0000 & 0.0000 & 0.0000 & 239 & 239 & 238 & 0.0000 & 0.0000 & 0.0001 \\
\hline 0 & 2 & 1 & 4 & 14 & 0.0000 & 0.0000 & 0.0000 & 7 & 13 & 63 & 0.0007 & 0.0023 & 0.0042 \\
\hline 1 & 2 & 8 & 8 & 21 & 0.0000 & 0.0000 & 0.0000 & 39 & 39 & 92 & 0.0002 & 0.0017 & 0.0064 \\
\hline 2 & 2 & 14 & 14 & 27 & 0.0000 & 0.0000 & 0.0000 & 72 & 72 & 121 & 0.0000 & 0.0006 & 0.0044 \\
\hline 3 & 2 & 21 & 21 & 34 & 0.0000 & 0.0000 & 0.0000 & 105 & 105 & 151 & 0.0000 & 0.0001 & 0.0023 \\
\hline 4 & 2 & 27 & 27 & 40 & 0.0000 & 0.0000 & 0.0000 & 139 & 139 & 180 & 0.0000 & 0.0000 & 0.0009 \\
\hline 5 & 2 & 34 & 34 & 47 & 0.0000 & 0.0000 & 0.0000 & 172 & 172 & 209 & 0.0000 & 0.0000 & 0.0002 \\
\hline 0 & 3 & 1 & 5 & 21 & 0.0000 & 0.0000 & 0.0000 & 7 & 14 & 92 & 0.0000 & 0.0001 & 0.0003 \\
\hline
\end{tabular}




\begin{tabular}{|rr|rrrrrr|rrrrrr|}
1 & 3 & 8 & 9 & 27 & 0.0000 & 0.0000 & 0.0000 & 39 & 39 & 121 & 0.0000 & 0.0001 & 0.0004 \\
2 & 3 & 14 & 14 & 34 & 0.0000 & 0.0000 & 0.0000 & 72 & 72 & 151 & 0.0000 & 0.0000 & 0.0003 \\
3 & 3 & 21 & 21 & 40 & 0.0000 & 0.0000 & 0.0000 & 105 & 105 & 180 & 0.0000 & 0.0000 & 0.0001 \\
\hline
\end{tabular}

For example, when $x_{s}=2$ and $x_{i}=0, M^{*}=14$ for all three estimators for 1-year data, while $M^{*}=72$ for exI and prI and $M^{*}=63$ for inI for 5-year data. The estimates are higher for 5-year data because they use a smaller realized, cumulative $g$ to account for mortality in the non-monitored years $2-4$. The probability of that occurrence $\left(x_{s}=2\right.$ and $x_{i}=0$ through 5 years) is 0.0311 when $\lambda=2$ and 0.1183 when $\lambda=5$ (Table 1 ).

When $\lambda \leq 10, M^{*}$ is the same for all three estimators after the first year over 98 percent of the time (top 6 rows of left panel in table). The cases where they differ are rare. For example, when $x_{s}=6$ and $x_{i}=0, M_{\mathrm{exI}}^{*}=M_{\mathrm{prI}}^{*}=41$ and $M_{\mathrm{inI}}^{*}=40$; the probability of that result is $<0.00005$ when $\lambda=2,0.00011$ when $\lambda=5$, and 0.0035 when $\lambda=10$ (Table 1 ).

When the year of formal monitoring is followed by four non-monitored years, $M_{\mathrm{exI}}^{*}=$ $M_{\mathrm{prI}}^{*}$ in all cases where either $x_{s}>0$ or $x_{i}=0$. These cases account for over 95 percent of the total. In other words, exI and prI would be in agreement more than 95 percent of the time when $\lambda \leq 10$ for 5 -year data and over 99 percent of the time for 1-year data (assuming $g_{i}=0.005$ and $\alpha=0.5$, as in table 2 ). The only practical distinction between $M_{\mathrm{exI}}^{*}$ and $M_{\mathrm{prI}}^{*}$ for 5-year data is when $x_{s}=0$ and $x_{i}=1$, in which case $M_{\mathrm{exI}}^{*}=7$ and $M_{\mathrm{prI}}^{*}=10$. This situation would occur 35 percent of the time for $\lambda=2,5$ or 10 . All other cases where $M_{\mathrm{exI}}^{*}$ and $M_{\mathrm{prI}}^{*}$ differ occur with frequency less than 1 percent combined (Table 1).

When no incidentals are found over the course of 5 years, $M_{\text {inI }}^{*}$ is consistently less than $M_{\mathrm{exI}}^{*}$ and $M_{\mathrm{prI}}^{*}$, and 95.7 percent of time inI will have a smaller $M^{*}$ than will prI or exI when $\lambda=2$. When $\lambda$ is much greater than 2 , the probability of finding incidentals increases significantly, as does the probability that $M_{\mathrm{inI}}^{*}>M_{\mathrm{prI}}^{*}$. For example, when $\lambda=5$, the probability that $M_{\mathrm{inI}}^{*}>M_{\mathrm{prI}}^{*}$ is 10.2 percent, and when $\lambda$ increases to $10, \operatorname{Pr}\left(M_{\mathrm{inI}}^{*}>M_{\mathrm{prI}}^{*}\right)$ rises to 19 percent (table 2 ).

Tables with all combinations of $g_{i} \in\{0.005,0.01,0.05\}$ and $\alpha \in\{0.5,0.1\}$ are presented below.

Table 2. $M^{*} \mid\left\{x_{s}, x_{i}\right\}$ for all three estimators, with $\alpha=0.5, g_{i}=0.01$.

\begin{tabular}{|c|c|c|c|c|c|c|c|c|c|c|c|c|c|}
\hline \multirow{3}{*}{$x_{s}$} & \multirow{3}{*}{$x_{i}$} & \multicolumn{6}{|c|}{1 year } & \multicolumn{6}{|c|}{5 years } \\
\hline & & \multirow{2}{*}{$\boldsymbol{M}_{\mathrm{exI}}^{*}$} & \multirow{2}{*}{$\boldsymbol{M}_{\mathrm{prI}}^{*}$} & \multirow{2}{*}{$\boldsymbol{M}_{\mathrm{inI}}^{*}$} & \multicolumn{3}{|c|}{$\operatorname{Pr}\left(x_{s}, x_{i} \mid \lambda, g_{i}=0.01\right)$} & \multirow{2}{*}{$\boldsymbol{M}_{\mathrm{exI}}^{*}$} & \multirow{2}{*}{$\boldsymbol{M}_{\mathrm{prI}}^{*}$} & \multirow{2}{*}{$\boldsymbol{M}_{\mathrm{inI}}^{*}$} & \multicolumn{3}{|c|}{$\operatorname{Pr}\left(x_{s}, x_{i} \mid \lambda, g_{i}=0.01\right)$} \\
\hline & & & & & $\lambda=2$ & $\lambda=\mathbf{5}$ & $\lambda=10$ & & & & $\lambda=2$ & $\lambda=\mathbf{5}$ & $\lambda=10$ \\
\hline 0 & 0 & 1 & 1 & 1 & 0.7359 & 0.4661 & 0.2169 & 7 & 7 & 5 & 0.6794 & 0.3814 & 0.1444 \\
\hline 1 & 0 & 8 & 8 & 7 & 0.2223 & 0.3491 & 0.3267 & 39 & 39 & 30 & 0.2055 & 0.2868 & 0.2192 \\
\hline 2 & 0 & 14 & 14 & 14 & 0.0331 & 0.1308 & 0.2431 & 72 & 72 & 56 & 0.0305 & 0.1077 & 0.1625 \\
\hline 3 & 0 & 21 & 21 & 20 & 0.0030 & 0.0327 & 0.1215 & 105 & 105 & 82 & 0.0027 & 0.0269 & 0.0803 \\
\hline 4 & 0 & 27 & 27 & 27 & 0.0002 & 0.0061 & 0.0455 & 139 & 139 & 108 & 0.0002 & 0.0050 & 0.0303 \\
\hline 5 & 0 & 34 & 34 & 34 & 0.0000 & 0.0007 & 0.0139 & 172 & 172 & 134 & 0.0000 & 0.0006 & 0.0090 \\
\hline 6 & 0 & 41 & 41 & 40 & 0.0000 & 0.0001 & 0.0033 & 205 & 205 & 160 & 0.0000 & 0.0001 & 0.0024 \\
\hline 7 & 0 & 47 & 47 & 47 & 0.0000 & 0.0000 & 0.0008 & 239 & 239 & 186 & 0.0000 & 0.0000 & 0.0006 \\
\hline 8 & 0 & 54 & 54 & 53 & 0.0000 & 0.0000 & 0.0001 & 272 & 272 & 211 & 0.0000 & 0.0000 & 0.0001 \\
\hline
\end{tabular}




\begin{tabular}{|ll|rrrrrr|rrrrrr}
0 & 1 & 1 & 3 & 7 & 0.0040 & 0.0066 & 0.0068 & 7 & 10 & 30 & 0.0581 & 0.0819 & 0.0641 \\
1 & 1 & 8 & 7 & 14 & 0.0013 & 0.0054 & 0.0092 & 39 & 39 & 56 & 0.0172 & 0.0609 & 0.0947 \\
2 & 1 & 14 & 14 & 20 & 0.0002 & 0.0018 & 0.0069 & 72 & 72 & 82 & 0.0027 & 0.0223 & 0.0709 \\
3 & 1 & 21 & 21 & 27 & 0.0000 & 0.0005 & 0.0031 & 105 & 105 & 108 & 0.0003 & 0.0058 & 0.0357 \\
4 & 1 & 27 & 27 & 34 & 0.0000 & 0.0001 & 0.0013 & 139 & 139 & 134 & 0.0000 & 0.0011 & 0.0130 \\
5 & 1 & 34 & 34 & 40 & 0.0000 & 0.0000 & 0.0005 & 172 & 172 & 160 & 0.0000 & 0.0001 & 0.0045 \\
6 & 1 & 41 & 41 & 47 & 0.0000 & 0.0000 & 0.0001 & 205 & 205 & 186 & 0.0000 & 0.0000 & 0.0008 \\
7 & 1 & 47 & 47 & 53 & 0.0000 & 0.0000 & 0.0000 & 239 & 239 & 211 & 0.0000 & 0.0000 & 0.0002 \\
0 & 2 & 1 & 4 & 14 & 0.0000 & 0.0001 & 0.0001 & 7 & 13 & 56 & 0.0024 & 0.0087 & 0.0133 \\
1 & 2 & 8 & 8 & 20 & 0.0000 & 0.0000 & 0.0001 & 39 & 39 & 82 & 0.0008 & 0.0064 & 0.0193 \\
2 & 2 & 14 & 14 & 27 & 0.0000 & 0.0000 & 0.0001 & 72 & 72 & 108 & 0.0001 & 0.0024 & 0.0144 \\
3 & 2 & 21 & 21 & 34 & 0.0000 & 0.0000 & 0.0000 & 105 & 105 & 134 & 0.0000 & 0.0005 & 0.0074 \\
4 & 2 & 27 & 27 & 40 & 0.0000 & 0.0000 & 0.0000 & 139 & 139 & 160 & 0.0000 & 0.0001 & 0.0031 \\
5 & 2 & 34 & 34 & 47 & 0.0000 & 0.0000 & 0.0000 & 172 & 172 & 186 & 0.0000 & 0.0000 & 0.0008 \\
6 & 2 & 41 & 41 & 53 & 0.0000 & 0.0000 & 0.0000 & 205 & 205 & 211 & 0.0000 & 0.0000 & 0.0002 \\
7 & 2 & 47 & 47 & 60 & 0.0000 & 0.0000 & 0.0000 & 239 & 239 & 237 & 0.0000 & 0.0000 & 0.0001 \\
0 & 3 & 1 & 5 & 20 & 0.0000 & 0.0000 & 0.0000 & 7 & 14 & 82 & 0.0001 & 0.0006 & 0.0019 \\
1 & 3 & 8 & 9 & 27 & 0.0000 & 0.0000 & 0.0000 & 39 & 39 & 108 & 0.0000 & 0.0004 & 0.0025 \\
2 & 3 & 14 & 14 & 34 & 0.0000 & 0.0000 & 0.0000 & 72 & 72 & 134 & 0.0000 & 0.0002 & 0.0021 \\
3 & 3 & 21 & 21 & 40 & 0.0000 & 0.0000 & 0.0000 & 105 & 105 & 160 & 0.0000 & 0.0000 & 0.0010 \\
4 & 3 & 27 & 27 & 47 & 0.0000 & 0.0000 & 0.0000 & 139 & 139 & 186 & 0.0000 & 0.0000 & 0.0004 \\
5 & 3 & 34 & 34 & 53 & 0.0000 & 0.0000 & 0.0000 & 172 & 172 & 211 & 0.0000 & 0.0000 & 0.0001 \\
0 & 4 & 1 & 7 & 27 & 0.0000 & 0.0000 & 0.0000 & 7 & 16 & 108 & 0.0000 & 0.0000 & 0.0001 \\
1 & 4 & 8 & 9 & 34 & 0.0000 & 0.0000 & 0.0000 & 39 & 40 & 134 & 0.0000 & 0.0000 & 0.0004 \\
2 & 4 & 14 & 14 & 40 & 0.0000 & 0.0000 & 0.0000 & 72 & 72 & 160 & 0.0000 & 0.0000 & 0.0002 \\
3 & 4 & 21 & 21 & 47 & 0.0000 & 0.0000 & 0.0000 & 105 & 105 & 186 & 0.0000 & 0.0000 & 0.0001 \\
\hline
\end{tabular}

Table 3. $M^{*} \mid\left\{x_{s}, x_{i}\right\}$ for all three estimators, with $\alpha=0.5, g_{i}=0.05$.

\begin{tabular}{|c|c|c|c|c|c|c|c|c|c|c|c|c|c|}
\hline \multirow{3}{*}{$x_{s}$} & \multirow{3}{*}{$x_{i}$} & \multicolumn{6}{|c|}{1 year } & \multicolumn{6}{|c|}{5 years } \\
\hline & & \multirow{2}{*}{$\boldsymbol{M}_{\mathrm{exI}}^{*}$} & \multirow{2}{*}{$\boldsymbol{M}_{\mathrm{prI}}^{*}$} & \multirow{2}{*}{$\boldsymbol{M}_{\mathrm{inI}}^{*}$} & \multicolumn{3}{|c|}{$\operatorname{Pr}\left(x_{s}, x_{i} \mid \lambda, g_{i}=0.05\right)$} & \multirow{2}{*}{$\boldsymbol{M}_{\mathrm{exI}}^{*}$} & \multirow{2}{*}{$\boldsymbol{M}_{\mathrm{prI}}^{*}$} & \multirow{2}{*}{$\boldsymbol{M}_{\mathrm{inI}}^{*}$} & \multicolumn{3}{|c|}{$\operatorname{Pr}\left(x_{s}, x_{i} \mid \lambda, g_{i}=0.05\right)$} \\
\hline & & & & & $\lambda=2$ & $\lambda=\mathbf{5}$ & $\lambda=10$ & & & & $\lambda=2$ & $\lambda=5$ & $\lambda=\mathbf{1 0}$ \\
\hline 0 & 0 & 1 & 1 & 1 & 0.7213 & 0.4413 & 0.1941 & 7 & 7 & 3 & 0.4835 & 0.1633 & 0.0256 \\
\hline 1 & 0 & 8 & 8 & 7 & 0.2156 & 0.3312 & 0.2888 & 39 & 39 & 16 & 0.1438 & 0.1215 & 0.0378 \\
\hline 2 & 0 & 14 & 14 & 13 & 0.0315 & 0.1224 & 0.2179 & 72 & 72 & 30 & 0.0211 & 0.0458 & 0.0292 \\
\hline 3 & 0 & 21 & 21 & 19 & 0.0038 & 0.0304 & 0.1101 & 105 & 105 & 43 & 0.0026 & 0.0117 & 0.0144 \\
\hline 4 & 0 & 27 & 27 & 25 & 0.0002 & 0.0060 & 0.0405 & 139 & 139 & 57 & 0.0001 & 0.0022 & 0.0053 \\
\hline 5 & 0 & 34 & 34 & 31 & 0.0000 & 0.0008 & 0.0120 & 172 & 172 & 71 & 0.0000 & 0.0003 & 0.0017 \\
\hline 6 & 0 & 41 & 41 & 37 & 0.0000 & 0.0001 & 0.0030 & 205 & 205 & 84 & 0.0000 & 0.0001 & 0.0004 \\
\hline 7 & 0 & 47 & 47 & 43 & 0.0000 & 0.0000 & 0.0006 & 239 & 239 & 98 & 0.0000 & 0.0000 & 0.0000 \\
\hline 8 & 0 & 54 & 54 & 49 & 0.0000 & 0.0000 & 0.0002 & 272 & 272 & 112 & 0.0000 & 0.0000 & 0.0000 \\
\hline 0 & 1 & 1 & 3 & 7 & 0.0204 & 0.0311 & 0.0273 & 7 & 10 & 16 & 0.2064 & 0.1728 & 0.0563 \\
\hline
\end{tabular}




\begin{tabular}{|c|c|c|c|c|c|c|c|c|c|c|c|c|c|}
\hline 1 & 1 & 8 & 7 & 13 & 0.0059 & 0.0226 & 0.0420 & 39 & 39 & 30 & 0.0628 & 0.1293 & 0.0845 \\
\hline 2 & 1 & 14 & 14 & 19 & 0.0008 & 0.0087 & 0.0303 & 72 & 72 & 43 & 0.0089 & 0.0480 & 0.0623 \\
\hline 3 & 1 & 21 & 21 & 25 & 0.0001 & 0.0022 & 0.0157 & 105 & 105 & 57 & 0.0010 & 0.0119 & 0.0323 \\
\hline 4 & 1 & 27 & 27 & 31 & 0.0000 & 0.0004 & 0.0058 & 139 & 139 & 71 & 0.0000 & 0.0025 & 0.0118 \\
\hline 5 & 1 & 34 & 34 & 37 & 0.0000 & 0.0001 & 0.0020 & 172 & 172 & 84 & 0.0000 & 0.0003 & 0.0036 \\
\hline 6 & 1 & 41 & 41 & 43 & 0.0000 & 0.0000 & 0.0005 & 205 & 205 & 98 & 0.0000 & 0.0000 & 0.0010 \\
\hline 7 & 1 & 47 & 47 & 49 & 0.0000 & 0.0000 & 0.0001 & 239 & 239 & 112 & 0.0000 & 0.0000 & 0.0003 \\
\hline 8 & 1 & 54 & 54 & 56 & 0.0000 & 0.0000 & 0.0000 & 272 & 272 & 126 & 0.0000 & 0.0000 & 0.0001 \\
\hline 0 & 2 & 1 & 4 & 13 & 0.0003 & 0.0012 & 0.0020 & 7 & 13 & 30 & 0.0446 & 0.0935 & 0.0606 \\
\hline 1 & 2 & 8 & 8 & 19 & 0.0001 & 0.0009 & 0.0028 & 39 & 39 & 43 & 0.0129 & 0.0704 & 0.0891 \\
\hline 2 & 2 & 14 & 14 & 25 & 0.0000 & 0.0003 & 0.0022 & 72 & 72 & 57 & 0.0020 & 0.0254 & 0.0685 \\
\hline 3 & 2 & 21 & 21 & 31 & 0.0000 & 0.0001 & 0.0011 & 105 & 105 & 71 & 0.0002 & 0.0060 & 0.0347 \\
\hline 4 & 2 & 27 & 27 & 37 & 0.0000 & 0.0000 & 0.0003 & 139 & 139 & 84 & 0.0000 & 0.0012 & 0.0123 \\
\hline 5 & 2 & 34 & 34 & 43 & 0.0000 & 0.0000 & 0.0001 & 172 & 172 & 98 & 0.0000 & 0.0002 & 0.0039 \\
\hline 6 & 2 & 41 & 41 & 49 & 0.0000 & 0.0000 & 0.0000 & 205 & 205 & 112 & 0.0000 & 0.0000 & 0.0010 \\
\hline 7 & 2 & 47 & 47 & 56 & 0.0000 & 0.0000 & 0.0000 & 239 & 239 & 126 & 0.0000 & 0.0000 & 0.0002 \\
\hline 8 & 2 & 54 & 54 & 62 & 0.0000 & 0.0000 & 0.0000 & 272 & 272 & 139 & 0.0000 & 0.0000 & 0.0001 \\
\hline 0 & 3 & 1 & 5 & 19 & 0.0000 & 0.0000 & 0.0001 & 7 & 14 & 43 & 0.0066 & 0.0336 & 0.0426 \\
\hline 1 & 3 & 8 & 9 & 25 & 0.0000 & 0.0000 & 0.0002 & 39 & 39 & 57 & 0.0018 & 0.0251 & 0.0660 \\
\hline 2 & 3 & 14 & 14 & 31 & 0.0000 & 0.0000 & 0.0001 & 72 & 72 & 71 & 0.0003 & 0.0090 & 0.0478 \\
\hline 3 & 3 & 21 & 21 & 37 & 0.0000 & 0.0000 & 0.0001 & 105 & 105 & 84 & 0.0000 & 0.0022 & 0.0243 \\
\hline 4 & 3 & 27 & 27 & 43 & 0.0000 & 0.0000 & 0.0000 & 139 & 139 & 98 & 0.0000 & 0.0004 & 0.0094 \\
\hline 5 & 3 & 34 & 34 & 49 & 0.0000 & 0.0000 & 0.0000 & 172 & 172 & 112 & 0.0000 & 0.0000 & 0.0026 \\
\hline 6 & 3 & 41 & 41 & 56 & 0.0000 & 0.0000 & 0.0000 & 205 & 205 & 126 & 0.0000 & 0.0000 & 0.0006 \\
\hline 7 & 3 & 47 & 47 & 62 & 0.0000 & 0.0000 & 0.0000 & 239 & 239 & 139 & 0.0000 & 0.0000 & 0.0002 \\
\hline 0 & 4 & 1 & 7 & 25 & 0.0000 & 0.0000 & 0.0000 & 7 & 16 & 57 & 0.0009 & 0.0083 & 0.0234 \\
\hline 1 & 4 & 8 & 9 & 31 & 0.0000 & 0.0000 & 0.0000 & 39 & 40 & 71 & 0.0003 & 0.0068 & 0.0342 \\
\hline 2 & 4 & 14 & 14 & 37 & 0.0000 & 0.0000 & 0.0000 & 72 & 72 & 84 & 0.0000 & 0.0026 & 0.0257 \\
\hline 3 & 4 & 21 & 21 & 43 & 0.0000 & 0.0000 & 0.0000 & 105 & 105 & 98 & 0.0000 & 0.0006 & 0.0130 \\
\hline 4 & 4 & 27 & 27 & 49 & 0.0000 & 0.0000 & 0.0000 & 139 & 139 & 112 & 0.0000 & 0.0002 & 0.0050 \\
\hline 5 & 4 & 34 & 34 & 56 & 0.0000 & 0.0000 & 0.0000 & 172 & 172 & 126 & 0.0000 & 0.0000 & 0.0014 \\
\hline 6 & 4 & 41 & 41 & 62 & 0.0000 & 0.0000 & 0.0000 & 205 & 205 & 139 & 0.0000 & 0.0000 & 0.0003 \\
\hline 7 & 4 & 47 & 47 & 68 & 0.0000 & 0.0000 & 0.0000 & 239 & 239 & 153 & 0.0000 & 0.0000 & 0.0001 \\
\hline 0 & 5 & 1 & 8 & 31 & 0.0000 & 0.0000 & 0.0000 & 7 & 18 & 71 & 0.0000 & 0.0018 & 0.0102 \\
\hline 1 & 5 & 8 & 10 & 37 & 0.0000 & 0.0000 & 0.0000 & 39 & 40 & 84 & 0.0000 & 0.0014 & 0.0147 \\
\hline 2 & 5 & 14 & 15 & 43 & 0.0000 & 0.0000 & 0.0000 & 72 & 72 & 98 & 0.0000 & 0.0005 & 0.0115 \\
\hline 3 & 5 & 21 & 21 & 49 & 0.0000 & 0.0000 & 0.0000 & 105 & 105 & 112 & 0.0000 & 0.0002 & 0.0053 \\
\hline 4 & 5 & 27 & 27 & 56 & 0.0000 & 0.0000 & 0.0000 & 139 & 139 & 126 & 0.0000 & 0.0000 & 0.0022 \\
\hline 5 & 5 & 34 & 34 & 62 & 0.0000 & 0.0000 & 0.0000 & 172 & 172 & 139 & 0.0000 & 0.0000 & 0.0005 \\
\hline 6 & 5 & 41 & 41 & 68 & 0.0000 & 0.0000 & 0.0000 & 205 & 205 & 153 & 0.0000 & 0.0000 & 0.0002 \\
\hline 0 & 6 & 1 & 9 & 37 & 0.0000 & 0.0000 & 0.0000 & 7 & 19 & 84 & 0.0000 & 0.0003 & 0.0035 \\
\hline 1 & 6 & 8 & 11 & 43 & 0.0000 & 0.0000 & 0.0000 & 39 & 41 & 98 & 0.0000 & 0.0002 & 0.0053 \\
\hline 2 & 6 & 14 & 15 & 49 & 0.0000 & 0.0000 & 0.0000 & 72 & 72 & 112 & 0.0000 & 0.0002 & 0.0039 \\
\hline
\end{tabular}




\begin{tabular}{|ll|rrrrrr|rrrrrr|}
3 & 6 & 21 & 21 & 56 & 0.0000 & 0.0000 & 0.0000 & 105 & 105 & 126 & 0.0000 & 0.0000 & 0.0021 \\
4 & 6 & 27 & 27 & 62 & 0.0000 & 0.0000 & 0.0000 & 139 & 139 & 139 & 0.0000 & 0.0000 & 0.0006 \\
5 & 6 & 34 & 34 & 68 & 0.0000 & 0.0000 & 0.0000 & 172 & 172 & 153 & 0.0000 & 0.0000 & 0.0002 \\
0 & 7 & 1 & 10 & 43 & 0.0000 & 0.0000 & 0.0000 & 7 & 20 & 98 & 0.0000 & 0.0001 & 0.0010 \\
1 & 7 & 8 & 12 & 49 & 0.0000 & 0.0000 & 0.0000 & 39 & 41 & 112 & 0.0000 & 0.0000 & 0.0017 \\
2 & 7 & 14 & 16 & 56 & 0.0000 & 0.0000 & 0.0000 & 72 & 72 & 126 & 0.0000 & 0.0000 & 0.0012 \\
3 & 7 & 21 & 21 & 62 & 0.0000 & 0.0000 & 0.0000 & 105 & 105 & 139 & 0.0000 & 0.0000 & 0.0006 \\
4 & 7 & 27 & 27 & 68 & 0.0000 & 0.0000 & 0.0000 & 139 & 139 & 153 & 0.0000 & 0.0000 & 0.0002 \\
5 & 7 & 34 & 34 & 74 & 0.0000 & 0.0000 & 0.0000 & 172 & 172 & 167 & 0.0000 & 0.0000 & 0.0001 \\
0 & 8 & 1 & 11 & 49 & 0.0000 & 0.0000 & 0.0000 & 7 & 22 & 112 & 0.0000 & 0.0000 & 0.0004 \\
1 & 8 & 8 & 13 & 56 & 0.0000 & 0.0000 & 0.0000 & 39 & 42 & 126 & 0.0000 & 0.0000 & 0.0003 \\
2 & 8 & 14 & 16 & 62 & 0.0000 & 0.0000 & 0.0000 & 72 & 72 & 139 & 0.0000 & 0.0000 & 0.0004 \\
3 & 8 & 21 & 21 & 68 & 0.0000 & 0.0000 & 0.0000 & 105 & 105 & 153 & 0.0000 & 0.0000 & 0.0001 \\
0 & 9 & 1 & 12 & 56 & 0.0000 & 0.0000 & 0.0000 & 7 & 23 & 126 & 0.0000 & 0.0000 & 0.0001 \\
1 & 9 & 8 & 14 & 62 & 0.0000 & 0.0000 & 0.0000 & 39 & 43 & 139 & 0.0000 & 0.0000 & 0.0002 \\
3 & 9 & 21 & 22 & 74 & 0.0000 & 0.0000 & 0.0000 & 105 & 105 & 167 & 0.0000 & 0.0000 & 0.0001 \\
\hline
\end{tabular}

Table 4. $M^{*} \mid\left\{x_{s}, x_{i}\right\}$ for all three estimators, with $\alpha=0.1, g_{i}=0.005$.

\begin{tabular}{|c|c|c|c|c|c|c|c|c|c|c|c|c|c|}
\hline \multirow{3}{*}{$x_{s}$} & \multirow{3}{*}{$x_{i}$} & \multicolumn{6}{|c|}{1 year } & \multicolumn{6}{|c|}{5 years } \\
\hline & & \multirow{2}{*}{$\boldsymbol{M}_{\mathrm{exI}}^{*}$} & \multirow{2}{*}{$\boldsymbol{M}_{\mathrm{prI}}^{*}$} & \multirow{2}{*}{$\boldsymbol{M}_{\mathrm{inI}}^{*}$} & \multicolumn{3}{|c|}{$\operatorname{Pr}\left(x_{s}, x_{i} \mid \lambda, g_{i}=0.005\right)$} & \multirow{2}{*}{$\boldsymbol{M}_{\mathrm{exI}}^{*}$} & \multirow{2}{*}{$\boldsymbol{M}_{\mathrm{prI}}^{*}$} & \multirow{2}{*}{$\boldsymbol{M}_{\mathrm{inI}}^{*}$} & \multicolumn{3}{|c|}{$\operatorname{Pr}\left(x_{s}, x_{i} \mid \lambda, g_{i}=0.005\right)$} \\
\hline & & & & & $\lambda=2$ & $\lambda=\mathbf{5}$ & $\lambda=\mathbf{1 0}$ & & & & $\lambda=2$ & $\lambda=\mathbf{5}$ & $\lambda=\mathbf{1 0}$ \\
\hline 0 & 0 & 8 & 8 & 8 & 0.7396 & 0.4689 & 0.2202 & 44 & 44 & 38 & 0.7103 & 0.4244 & 0.1808 \\
\hline 1 & 0 & 20 & 20 & 19 & 0.2212 & 0.3517 & 0.3271 & 103 & 103 & 90 & 0.2129 & 0.3179 & 0.2686 \\
\hline 2 & 0 & 29 & 29 & 29 & 0.0326 & 0.1317 & 0.2490 & 152 & 152 & 133 & 0.0315 & 0.1193 & 0.2041 \\
\hline 3 & 0 & 38 & 38 & 38 & 0.0033 & 0.0330 & 0.1239 & 199 & 199 & 174 & 0.0032 & 0.0299 & 0.1016 \\
\hline 4 & 0 & 47 & 47 & 47 & 0.0002 & 0.0065 & 0.0467 & 243 & 243 & 212 & 0.0002 & 0.0060 & 0.0386 \\
\hline 5 & 0 & 55 & 55 & 55 & 0.0000 & 0.0010 & 0.0148 & 286 & 286 & 250 & 0.0000 & 0.0009 & 0.0123 \\
\hline 6 & 0 & 64 & 64 & 63 & 0.0000 & 0.0001 & 0.0036 & 328 & 328 & 287 & 0.0000 & 0.0001 & 0.0029 \\
\hline 7 & 0 & 72 & 72 & 71 & 0.0000 & 0.0000 & 0.0008 & 369 & 369 & 323 & 0.0000 & 0.0000 & 0.0006 \\
\hline 8 & 0 & 80 & 80 & 79 & 0.0000 & 0.0000 & 0.0001 & 410 & 410 & 359 & 0.0000 & 0.0000 & 0.0001 \\
\hline 0 & 1 & 0 & 10 & 19 & 0.0 & 0.0 & 32 & 44 & 48 & 90 & 07 & 0.0455 & 0.0380 \\
\hline 1 & 1 & 20 & 19 & 29 & 0.0006 & 0.0024 & 0.0048 & 103 & 103 & 133 & 0.0086 & 0.0344 & 0.0569 \\
\hline 2 & 1 & 29 & 29 & 38 & 0.0001 & 0.0008 & 0.0033 & 152 & 152 & 174 & 0.0012 & 0.0126 & 0.0434 \\
\hline 3 & 1 & 38 & 38 & 47 & 0.0000 & 0.0002 & 0.0017 & 199 & 198 & 212 & 0.0001 & 0.0030 & 0.0216 \\
\hline 4 & 1 & 47 & 47 & 55 & 0.0000 & 0.0001 & 0.0005 & 243 & 243 & 250 & 0.0000 & 0.0006 & 0.0077 \\
\hline 5 & 1 & 55 & 55 & 63 & 0.0000 & 0.0000 & 0.0002 & 286 & 286 & 287 & 0.0000 & 0.0001 & 0.0024 \\
\hline 6 & 1 & 64 & 64 & 71 & 0.0000 & 0.0000 & 0.0001 & 328 & 328 & 323 & 0.0000 & 0.0000 & 0.0007 \\
\hline 7 & 1 & 72 & 72 & 79 & 0.0000 & 0.0000 & 0.0000 & 369 & 369 & 359 & 0.0000 & 0.0000 & 0.0002 \\
\hline 0 & 2 & 8 & 12 & 29 & 0.0000 & 0.0000 & 0.0000 & 44 & 52 & 133 & 0.0008 & 0.0025 & 0.0043 \\
\hline 1 & 2 & 20 & 20 & 38 & 0.0000 & 0.0000 & 0.0000 & 103 & 103 & 174 & 0.0002 & 0.0019 & 0.0059 \\
\hline 2 & 2 & 29 & 29 & 47 & 0.0000 & 0.0000 & 0.0000 & 152 & 152 & 212 & 0.0000 & 0.0006 & 0.0047 \\
\hline
\end{tabular}




\begin{tabular}{|rl|rrrrrr|rrrrrr|}
3 & 2 & 38 & 38 & 55 & 0.0000 & 0.0000 & 0.0000 & 199 & 198 & 250 & 0.0000 & 0.0002 & 0.0023 \\
4 & 2 & 47 & 47 & 63 & 0.0000 & 0.0000 & 0.0000 & 243 & 243 & 287 & 0.0000 & 0.0000 & 0.0008 \\
5 & 2 & 55 & 55 & 71 & 0.0000 & 0.0000 & 0.0000 & 286 & 286 & 323 & 0.0000 & 0.0000 & 0.0003 \\
6 & 2 & 64 & 64 & 79 & 0.0000 & 0.0000 & 0.0000 & 328 & 328 & 359 & 0.0000 & 0.0000 & 0.0001 \\
0 & 3 & 8 & 13 & 38 & 0.0000 & 0.0000 & 0.0000 & 44 & 54 & 174 & 0.0000 & 0.0000 & 0.0003 \\
1 & 3 & 20 & 20 & 47 & 0.0000 & 0.0000 & 0.0000 & 103 & 103 & 212 & 0.0000 & 0.0000 & 0.0005 \\
2 & 3 & 29 & 29 & 55 & 0.0000 & 0.0000 & 0.0000 & 152 & 152 & 250 & 0.0000 & 0.0000 & 0.0003 \\
3 & 3 & 38 & 38 & 63 & 0.0000 & 0.0000 & 0.0000 & 199 & 198 & 287 & 0.0000 & 0.0000 & 0.0002 \\
\hline
\end{tabular}


Table 5. $M^{*} \mid\left\{x_{s}, x_{i}\right\}$ for all three estimators, with $\alpha=0.1, g_{i}=0.01$.

\begin{tabular}{|c|c|c|c|c|c|c|c|c|c|c|c|c|c|}
\hline \multirow{3}{*}{$x_{s}$} & \multirow{3}{*}{$x_{i}$} & \multicolumn{6}{|c|}{1 year } & \multicolumn{6}{|c|}{5 years } \\
\hline & & \multirow{2}{*}{$\boldsymbol{M}_{\mathrm{exI}}^{*}$} & \multirow{2}{*}{$\boldsymbol{M}_{\mathrm{prI}}^{*}$} & \multirow{2}{*}{$\boldsymbol{M}_{\mathrm{inI}}^{*}$} & \multicolumn{3}{|c|}{$\operatorname{Pr}\left(x_{s}, x_{i} \mid \lambda, g_{i}=0.01\right)$} & \multirow{2}{*}{$\boldsymbol{M}_{\mathrm{exI}}^{*}$} & \multirow{2}{*}{$\boldsymbol{M}_{\mathrm{prI}}^{*}$} & \multirow{2}{*}{$\boldsymbol{M}_{\mathrm{inI}}^{*}$} & \multicolumn{3}{|c|}{$\operatorname{Pr}\left(x_{s}, x_{i} \mid \lambda, g_{i}=0.01\right)$} \\
\hline & & & & & $\lambda=2$ & $\lambda=\mathbf{5}$ & $\lambda=\mathbf{1 0}$ & & & & $\lambda=2$ & $\lambda=\mathbf{5}$ & $\lambda=10$ \\
\hline 0 & 0 & 8 & 8 & 8 & 0.7379 & 0.4640 & 0.2176 & 44 & 44 & 34 & 0.6813 & 0.3786 & 0.1457 \\
\hline 1 & 0 & 20 & 20 & 19 & 0.2202 & 0.3503 & 0.3270 & 103 & 103 & 80 & 0.2033 & 0.2872 & 0.2196 \\
\hline 2 & 0 & 29 & 29 & 29 & 0.0324 & 0.1319 & 0.2437 & 152 & 152 & 118 & 0.0302 & 0.1084 & 0.1637 \\
\hline 3 & 0 & 38 & 38 & 38 & 0.0033 & 0.0326 & 0.1201 & 199 & 199 & 154 & 0.0030 & 0.0267 & 0.0809 \\
\hline 4 & 0 & 47 & 47 & 46 & 0.0003 & 0.0063 & 0.0456 & 243 & 243 & 188 & 0.0003 & 0.0051 & 0.0306 \\
\hline 5 & 0 & 55 & 55 & 54 & 0.0000 & 0.0010 & 0.0137 & 286 & 286 & 222 & 0.0000 & 0.0008 & 0.0092 \\
\hline 6 & 0 & 64 & 64 & 63 & 0.0000 & 0.0001 & 0.0038 & 328 & 328 & 255 & 0.0000 & 0.0001 & 0.0025 \\
\hline 7 & 0 & 72 & 72 & 71 & 0.0000 & 0.0000 & 0.0008 & 369 & 369 & 287 & 0.0000 & 0.0000 & 0.0005 \\
\hline 8 & 0 & 80 & 80 & 79 & 0.0000 & 0.0000 & 0.0001 & 410 & 410 & 319 & 0.0000 & 0.0000 & 0.0001 \\
\hline 0 & 1 & 8 & 10 & 19 & 0.0043 & 0.0067 & 0.0060 & 44 & 48 & 80 & 0.0584 & 0.0825 & 0.0626 \\
\hline 1 & 1 & 20 & 19 & 29 & 0.0014 & 0.0046 & 0.0094 & 103 & 103 & 118 & 0.0175 & 0.0611 & 0.0933 \\
\hline 2 & 1 & 29 & 29 & 38 & 0.0001 & 0.0019 & 0.0067 & 152 & 152 & 154 & 0.0023 & 0.0228 & 0.0699 \\
\hline 3 & 1 & 38 & 38 & 46 & 0.0000 & 0.0005 & 0.0035 & 199 & 198 & 188 & 0.0003 & 0.0058 & 0.0345 \\
\hline 4 & 1 & 47 & 47 & 54 & 0.0000 & 0.0001 & 0.0013 & 243 & 243 & 222 & 0.0000 & 0.0011 & 0.0130 \\
\hline 5 & 1 & 5 & 5 & 63 & 0.0000 & 0.0000 & 0.0004 & 286 & 286 & 255 & 0.0000 & 0.0001 & 0.0041 \\
\hline 6 & 1 & 64 & 64 & 71 & 0.0000 & 0.0000 & 0.0000 & 328 & 328 & 287 & 0.0000 & 0.0000 & 0.0011 \\
\hline 7 & 1 & 72 & 72 & 79 & 0.0000 & 0.0000 & 0.0000 & 369 & 369 & 319 & 0.0000 & 0.0000 & 0.0002 \\
\hline 0 & 2 & 8 & 12 & 29 & 0.0000 & 0.0000 & 0.0001 & 44 & 52 & 118 & 0.0025 & 0.0089 & 0.0135 \\
\hline 1 & 2 & 20 & 20 & 38 & 0.0000 & 0.0000 & 0.0001 & 103 & 103 & 154 & 0.0008 & 0.0063 & 0.0204 \\
\hline 2 & 2 & 29 & 29 & 46 & 0.0000 & 0.0000 & 0.0002 & 152 & 152 & 188 & 0.0001 & 0.0025 & 0.0148 \\
\hline 3 & 2 & 38 & 38 & 54 & 0.0000 & 0.0000 & 0.0001 & 199 & 198 & 222 & 0.0000 & 0.0006 & 0.0073 \\
\hline 4 & 2 & 47 & 47 & 63 & 0.0000 & 0.0000 & 0.0000 & 243 & 243 & 255 & 0.0000 & 0.0001 & 0.0030 \\
\hline 5 & 2 & 55 & 55 & 71 & 0.0000 & 0.0000 & 0.0000 & 286 & 286 & 287 & 0.0000 & 0.0000 & 0.0007 \\
\hline 6 & 2 & 64 & 64 & 79 & 0.0000 & 0.0000 & 0.0000 & 328 & 328 & 319 & 0.0000 & 0.0000 & 0.0002 \\
\hline 7 & 2 & 72 & 72 & 86 & 0.0000 & 0.0000 & 0.0000 & 369 & 369 & 350 & 0.0000 & 0.0000 & 0.0001 \\
\hline 0 & 3 & 8 & 13 & 38 & 0.0000 & 0.0000 & 0.0000 & 44 & 54 & 154 & 0.0001 & 0.0007 & 0.0018 \\
\hline 1 & 3 & 20 & 20 & 46 & 0.0000 & 0.0000 & 0.0000 & 103 & 103 & 188 & 0.0000 & 0.0004 & 0.0028 \\
\hline 2 & 3 & 29 & 29 & 54 & 0.0000 & 0.0000 & 0.0000 & 152 & 152 & 222 & 0.0000 & 0.0001 & 0.0021 \\
\hline 3 & 3 & 38 & 38 & 63 & 0.0000 & 0.0000 & 0.0000 & 199 & 198 & 255 & 0.0000 & 0.0001 & 0.0008 \\
\hline 4 & 3 & 47 & 47 & 71 & 0.0000 & 0.0000 & 0.0000 & 243 & 243 & 287 & 0.0000 & 0.0000 & 0.0003 \\
\hline 5 & 3 & 55 & 55 & 79 & 0.0000 & 0.0000 & 0.0000 & 286 & 286 & 319 & 0.0000 & 0.0000 & 0.0001 \\
\hline 0 & 4 & 8 & 15 & 46 & 0.0000 & 0.0000 & 0.0000 & 44 & 56 & 188 & 0.0000 & 0.0000 & 0.0002 \\
\hline 1 & 4 & 20 & 21 & 54 & 0.0000 & 0.0000 & 0.0000 & 103 & 104 & 222 & 0.0000 & 0.0000 & 0.0003 \\
\hline 2 & 4 & 29 & 29 & 63 & 0.0000 & 0.0000 & 0.0000 & 152 & 152 & 255 & 0.0000 & 0.0000 & 0.0002 \\
\hline 3 & 4 & 38 & 38 & 71 & 0.0000 & 0.0000 & 0.0000 & 199 & 198 & 287 & 0.0000 & 0.0000 & 0.0001 \\
\hline
\end{tabular}


Table 6. $M^{*} \mid\left\{x_{s}, x_{i}\right\}$ for all three estimators, with $\alpha=0.1, g_{i}=0.05$.

\begin{tabular}{|c|c|c|c|c|c|c|c|c|c|c|c|c|c|}
\hline \multirow{3}{*}{$x_{s}$} & & \multicolumn{6}{|c|}{1 year } & \multicolumn{6}{|c|}{5 years } \\
\hline & & \multirow{2}{*}{$\boldsymbol{M}_{\mathrm{exI}}^{*}$} & \multirow{2}{*}{$\boldsymbol{M}_{\mathrm{prI}}^{*}$} & \multirow{2}{*}{$\boldsymbol{M}_{\mathrm{inI}}^{*}$} & \multicolumn{3}{|c|}{$\operatorname{Pr}\left(x_{s}, x_{i} \mid \lambda, g_{i}=0.05\right)$} & \multirow{2}{*}{$\boldsymbol{M}_{\mathrm{exI}}^{*}$} & \multirow{2}{*}{$\boldsymbol{M}_{\mathrm{prI}}^{*}$} & \multirow{2}{*}{$\boldsymbol{M}_{\mathrm{inI}}^{*}$} & \multicolumn{3}{|c|}{$\operatorname{Pr}\left(x_{s}, x_{i} \mid \lambda, g_{i}=0.05\right)$} \\
\hline & & & & & $\lambda=2$ & $\lambda=\mathbf{5}$ & $\lambda=\mathbf{1 0}$ & & & & $\lambda=2$ & $\lambda=\mathbf{5}$ & $\lambda=\mathbf{1 0}$ \\
\hline 0 & 0 & 8 & 8 & 7 & 0.7181 & 0.4422 & 0.1915 & 44 & 44 & 17 & 0.4808 & 0.1629 & 0.0266 \\
\hline 1 & 0 & 20 & 20 & 18 & 0.2170 & 0.3298 & 0.2916 & 103 & 103 & 42 & 0.1471 & 0.1224 & 0.0405 \\
\hline 2 & 0 & 29 & 29 & 27 & 0.0326 & 0.1218 & 0.2176 & 152 & 152 & 62 & 0.0218 & 0.0446 & 0.0283 \\
\hline 3 & 0 & 38 & 38 & 35 & 0.0034 & 0.0305 & 0.1093 & 199 & 199 & 81 & 0.0023 & 0.0113 & 0.0150 \\
\hline 4 & 0 & 47 & 47 & 43 & 0.0002 & 0.0058 & 0.0413 & 243 & 243 & 99 & 0.0002 & 0.0021 & 0.0059 \\
\hline 5 & 0 & 55 & 55 & 50 & 0.0000 & 0.0008 & 0.0120 & 286 & 286 & 117 & 0.0000 & 0.0002 & 0.0017 \\
\hline 6 & 0 & 64 & 64 & 58 & 0.0000 & 0.0001 & 0.0031 & 328 & 328 & 134 & 0.0000 & 0.0000 & 0.0004 \\
\hline 7 & 0 & 72 & 72 & 66 & 0.0000 & 0.0000 & 0.0007 & 369 & 369 & 151 & 0.0000 & 0.0000 & 0.0001 \\
\hline 8 & 0 & 80 & 80 & 73 & 0.0000 & 0.0000 & 0.0001 & 410 & 410 & 168 & 0.0000 & 0.0000 & 0.0000 \\
\hline 0 & 1 & 8 & 10 & 18 & 0.0211 & 0.0319 & 0.0267 & 44 & 48 & 42 & 0.2066 & 0.1748 & 0.0549 \\
\hline 1 & 1 & 20 & 19 & 27 & 0.0061 & 0.0229 & 0.0416 & 103 & 103 & 62 & 0.0609 & 0.1284 & 0.0844 \\
\hline 2 & 1 & 29 & 29 & 35 & 0.0009 & 0.0086 & 0.0316 & 152 & 152 & 81 & 0.0095 & 0.0478 & 0.0633 \\
\hline 3 & 1 & 38 & 38 & 43 & 0.0001 & 0.0024 & 0.0156 & 199 & 198 & 99 & 0.0010 & 0.0120 & 0.0315 \\
\hline 4 & 1 & 47 & 47 & 50 & 0.0000 & 0.0005 & 0.0058 & 243 & 243 & 117 & 0.0000 & 0.0022 & 0.0114 \\
\hline 5 & 1 & 55 & 55 & 58 & 0.0000 & 0.0001 & 0.0018 & 286 & 286 & 134 & 0.0000 & 0.0003 & 0.0036 \\
\hline 6 & 1 & 64 & 64 & 66 & 0.0000 & 0.0000 & 0.0004 & 328 & 328 & 151 & 0.0000 & 0.0000 & 0.0011 \\
\hline 7 & 1 & 72 & 72 & 73 & 0.0000 & 0.0000 & 0.0001 & 369 & 369 & 168 & 0.0000 & 0.0000 & 0.0003 \\
\hline 0 & 2 & 8 & 12 & 27 & 0.0003 & 0.0011 & 0.0021 & 44 & 52 & 62 & 0.0450 & 0.0932 & 0.0600 \\
\hline 1 & 2 & 20 & 20 & 35 & 0.0001 & 0.0010 & 0.0026 & 103 & 103 & 81 & 0.0132 & 0.0702 & 0.0909 \\
\hline 2 & 2 & 29 & 29 & 43 & 0.0000 & 0.0003 & 0.0021 & 152 & 152 & 99 & 0.0019 & 0.0257 & 0.0686 \\
\hline 3 & 2 & 38 & 38 & 50 & 0.0000 & 0.0000 & 0.0011 & 199 & 198 & 117 & 0.0001 & 0.0064 & 0.0331 \\
\hline 4 & 2 & 47 & 47 & 58 & 0.0000 & 0.0000 & 0.0004 & 243 & 243 & 134 & 0.0000 & 0.0013 & 0.0123 \\
\hline 5 & 2 & 55 & 55 & 66 & 0.0000 & 0.0000 & 0.0002 & 286 & 286 & 151 & 0.0000 & 0.0002 & 0.0036 \\
\hline 6 & 2 & 64 & 64 & 73 & 0.0000 & 0.0000 & 0.0000 & 328 & 328 & 168 & 0.0000 & 0.0000 & 0.0009 \\
\hline 7 & 2 & 72 & 72 & 80 & 0.0000 & 0.0000 & 0.0000 & 369 & 369 & 184 & 0.0000 & 0.0000 & 0.0003 \\
\hline 0 & 3 & 8 & 13 & 35 & 0.0000 & 0.0000 & 0.0001 & 44 & 54 & 81 & 0.0064 & 0.0330 & 0.0420 \\
\hline 1 & 3 & 20 & 20 & 43 & 0.0000 & 0.0000 & 0.0002 & 103 & 103 & 99 & 0.0018 & 0.0245 & 0.0628 \\
\hline 2 & 3 & 29 & 29 & 50 & 0.0000 & 0.0000 & 0.0002 & 152 & 152 & 117 & 0.0003 & 0.0096 & 0.0487 \\
\hline 3 & 3 & 38 & 38 & 58 & 0.0000 & 0.0000 & 0.0000 & 199 & 198 & 134 & 0.0000 & 0.0024 & 0.0248 \\
\hline 4 & 3 & 47 & 47 & 66 & 0.0000 & 0.0000 & 0.0000 & 243 & 243 & 151 & 0.0000 & 0.0005 & 0.0094 \\
\hline 5 & 3 & 55 & 55 & 73 & 0.0000 & 0.0000 & 0.0000 & 286 & 286 & 168 & 0.0000 & 0.0001 & 0.0027 \\
\hline 6 & 3 & 64 & 64 & 80 & 0.0000 & 0.0000 & 0.0000 & 328 & 328 & 184 & 0.0000 & 0.0000 & 0.0006 \\
\hline 7 & 3 & 72 & 72 & 87 & 0.0000 & 0.0000 & 0.0000 & 369 & 369 & 201 & 0.0000 & 0.0000 & 0.0001 \\
\hline 0 & 4 & 8 & 15 & 43 & 0.0000 & 0.0000 & 0.0000 & 44 & 56 & 99 & 0.0008 & 0.0089 & 0.0222 \\
\hline 1 & 4 & 20 & 21 & 50 & 0.0000 & 0.0000 & 0.0000 & 103 & 104 & 117 & 0.0002 & 0.0065 & 0.0350 \\
\hline 2 & 4 & 29 & 29 & 58 & 0.0000 & 0.0000 & 0.0000 & 152 & 152 & 134 & 0.0000 & 0.0025 & 0.0253 \\
\hline 3 & 4 & 38 & 38 & 66 & 0.0000 & 0.0000 & 0.0000 & 199 & 198 & 151 & 0.0000 & 0.0007 & 0.0136 \\
\hline 4 & 4 & 47 & 47 & 73 & 0.0000 & 0.0000 & 0.0000 & 243 & 243 & 168 & 0.0000 & 0.0001 & 0.0049 \\
\hline
\end{tabular}




\begin{tabular}{|c|c|c|c|c|c|c|c|c|c|c|c|c|c|}
\hline 5 & 4 & 55 & 55 & 80 & 0.0000 & 0.0000 & 0.0000 & 286 & 286 & 184 & 0.0000 & 0.0000 & 0.0015 \\
\hline 0 & 4 & 64 & 64 & 87 & 0.0000 & 0.0000 & 0.0000 & 328 & 328 & 201 & 0.0000 & 0.0000 & 0.0004 \\
\hline & 4 & 72 & 72 & 95 & 0.0000 & 0.0000 & 0.0000 & 369 & 369 & 217 & 0.0000 & 0.0000 & 0.0001 \\
\hline 0 & 5 & 8 & 16 & 50 & 0.0000 & 0.0000 & 0.0000 & 44 & 58 & 117 & 0.0000 & 0.0019 & 0.0096 \\
\hline 1 & 5 & 20 & 22 & 58 & 0.0000 & 0.0000 & 0.0000 & 103 & 104 & 134 & 0.0000 & 0.0014 & 0.0152 \\
\hline 2 & 5 & 29 & 30 & 66 & 0.0000 & 0.0000 & 0.0000 & 152 & 152 & 151 & 0.0000 & 0.0005 & 0.0116 \\
\hline 3 & 5 & 38 & 38 & 73 & 0.0000 & 0.0000 & 0.0000 & 199 & 198 & 168 & 0.0000 & 0.0001 & 0.0054 \\
\hline 4 & 5 & 47 & 47 & 80 & 0.0000 & 0.0000 & 0.0000 & 243 & 243 & 184 & 0.0000 & 0.0000 & 0.0023 \\
\hline 5 & 5 & 55 & 55 & 87 & 0.0000 & 0.0000 & 0.0000 & 286 & 286 & 201 & 0.0000 & 0.0000 & 0.0006 \\
\hline 6 & 5 & 64 & 64 & 95 & 0.0000 & 0.0000 & 0.0000 & 328 & 328 & 217 & 0.0000 & 0.0000 & 0.0001 \\
\hline 0 & 6 & 8 & 17 & 58 & 0.0000 & 0.0000 & 0.0000 & 44 & 60 & 134 & 0.0000 & 0.0004 & 0.0036 \\
\hline 1 & 6 & 20 & 23 & 66 & 0.0000 & 0.0000 & 0.0000 & 103 & 104 & 151 & 0.0000 & 0.0002 & 0.0051 \\
\hline 2 & 6 & 29 & 30 & 73 & 0.0000 & 0.0000 & 0.0000 & 152 & 152 & 168 & 0.0000 & 0.0001 & 0.0040 \\
\hline 3 & 6 & 38 & 38 & 80 & 0.0000 & 0.0000 & 0.0000 & 199 & 198 & 184 & 0.0000 & 0.0000 & 0.0020 \\
\hline 4 & 6 & 47 & 47 & 87 & 0.0000 & 0.0000 & 0.0000 & 243 & 243 & 201 & 0.0000 & 0.0000 & 0.0009 \\
\hline 5 & 6 & 55 & 55 & 95 & 0.0000 & 0.0000 & 0.0000 & 286 & 286 & 217 & 0.0000 & 0.0000 & 0.0002 \\
\hline 6 & 6 & 64 & 64 & 102 & 0.0000 & 0.0000 & 0.0000 & 328 & 328 & 233 & 0.0000 & 0.0000 & 0.0001 \\
\hline 0 & 7 & 8 & 18 & 66 & 0.0000 & 0.0000 & 0.0000 & 44 & 62 & 151 & 0.0000 & 0.0001 & 0.0012 \\
\hline 1 & 7 & 20 & 23 & 73 & 0.0000 & 0.0000 & 0.0000 & 103 & 105 & 168 & 0.0000 & 0.0000 & 0.0017 \\
\hline 2 & 7 & 29 & 30 & 80 & 0.0000 & 0.0000 & 0.0000 & 152 & 152 & 184 & 0.0000 & 0.0000 & 0.0013 \\
\hline 3 & 7 & 38 & 38 & 87 & 0.0000 & 0.0000 & 0.0000 & 199 & 198 & 201 & 0.0000 & 0.0000 & 0.0006 \\
\hline 4 & 7 & 47 & 47 & 95 & 0.0000 & 0.0000 & 0.0000 & 243 & 243 & 217 & 0.0000 & 0.0000 & 0.0003 \\
\hline 0 & 8 & 8 & 19 & 73 & 0.0000 & 0.0000 & 0.0000 & 44 & 63 & 168 & 0.0000 & 0.0000 & 0.0003 \\
\hline 1 & 8 & 20 & 24 & 80 & 0.0000 & 0.0000 & 0.0000 & 103 & 105 & 184 & 0.0000 & 0.0000 & 0.0005 \\
\hline 2 & 8 & 29 & 31 & 87 & 0.0000 & 0.0000 & 0.0000 & 152 & 153 & 201 & 0.0000 & 0.0000 & 0.0003 \\
\hline 3 & 8 & 38 & 39 & 95 & 0.0000 & 0.0000 & 0.0000 & 199 & 198 & 217 & 0.0000 & 0.0000 & 0.0001 \\
\hline 4 & 8 & 47 & 47 & 102 & 0.0000 & 0.0000 & 0.0000 & 243 & 243 & 233 & 0.0000 & 0.0000 & 0.0001 \\
\hline 0 & 9 & 8 & 21 & 80 & 0.0000 & 0.0000 & 0.0000 & 44 & 65 & 184 & 0.0000 & 0.0000 & 0.0001 \\
\hline 1 & 9 & 20 & 25 & 87 & 0.0000 & 0.0000 & 0.0000 & 103 & 106 & 201 & 0.0000 & 0.0000 & 0.0002 \\
\hline 2 & 9 & 29 & 31 & 95 & 0.0000 & 0.0000 & 0.0000 & 152 & 153 & 217 & 0.0000 & 0.0000 & 0.0001 \\
\hline
\end{tabular}

\section{Conclusions}

All three estimators are statistically valid in the sense that they are rooted in probability theory and do not rely on wildly implausible assumptions. The exI (exclude incidentals) and prI (truncated prior) estimators produce virtually identical results, except in the somewhat unusual situation where $x_{s}=0$ and $x_{i}>0$, in which case $M_{\mathrm{prI}}^{*}$ tended to be greater than $M_{\mathrm{exI}}^{*}$. Both tended to overestimate when $\lambda$ was small and credibility level $(1-\alpha)$ was large, and both converged on nominal coverage at close to the same rate when $\alpha$ was 0.5 . inI, which includes incidentals in the observed carcass count and uses an assumed or estimated $g_{i}$, had similar or much better coverage than the other two when the detection probability in unmonitored years was correctly estimated (or assumed), but had poorer coverage properties when badly misspecified. However, if $g_{i}$ was "small" $(\leq 1 \%)$ and was assumed small, the coverage properties were still better than or comparable to coverages for exI and prI even when $g_{i}$ was 
misspecified (as, say, $g_{i}$ assumed to be $1 \%$ when it is really $0.1 \%$ ). These small $g_{i}$ values may not be applicable for eagles or other large raptors, which would require a separate analysis.

The probability of detection $(g)$ in EoA accounts for carcasses that occur outside of the study either in time (non-monitoring years) or space (outside of searched plots), so exI (exclude incidentals) in EoA is valid. Although valid, the statistical power of exI rapidly declines as an estimate of $g$ is diluted through non-monitored years. Additionally, if incidentals are found but are excluded from estimation of total mortality $\left(M^{*}\right)$, it is possible for the estimate of mortality to be less than the number of carcasses actually observed. Information available in incidental carcass discoveries can be incorporated into EoA by truncating the lower bound of the prior distribution for $M$ (prI) to ensure that $M^{*} \geq x$ and to improve on the statistical power of EoA. If the detection probability for incidentals $\left(g_{i}\right)$ can be estimated, incorporating $g_{i}$ into the overall estimation of $g$ and including the incidentals in the carcass count used for calculating $M^{*}$ would provide a more complete accounting of the information available in incidentals. This would have the effect of increasing the overall $g$ value, leading to lower mortality estimates in most cases and partially correcting for the tendency of exI and prI and to overestimate mortality when $\lambda$ is small. However, in the unusual event that $\lambda$ is small and incidentals are found in nonmonitored years, inI would substantially overestimate $M$. If $g_{i}$ is badly misspecified or poorly estimated, $M^{*}$ may be strongly biased. For larger species like eagles or other large raptors, accurate estimation of $g_{i}$ may be more crucial than it is for bats.

\section{Limitations}

\section{Frequencies, Not Magnitudes}

This modest simulation study is limited to exploration of basic statistical properties of three approaches to accounting for incidentals under some simplified scenarios. The primary focus is the coverage probabilities for estimating $M$ and the frequencies with which the estimators over- or under-estimate $M^{*}$. The magnitude of over-estimations is discussed only briefly, but the extensive results tables show $M^{*}$ values in a wide array of scenarios. These $M^{*}$ values should not be interpreted in isolation but only in conjunction with the probabilities with which they occur.

\section{Sensitivity and Specificity}

The frequencies with which high fatality rates are not detected (false negatives) and low fatality rates are mistakenly interpreted as high (false positives) are discussed only briefly. In a general framework of 1 year of monitoring followed by 4 non-monitored years, there is limited statistical power to distinguish clearly between high and low fatality rates. The difficulty is compounded by the high degree of volatility that is inherent in small count data. In addition, the simulations are based on a simplifying assumption that the true number of annual fatalities varies as a Poisson random variable with constant mean, but in practice the numbers of annual fatalities are likely to be more variable than Poisson or have rates that vary significantly from year to year. This would affect the coverage probabilities, increase the frequencies of very high $M^{*}$ 's, and increase the probabilities that high fatality rates would pass unnoticed in the non-monitored years. 


\section{Alternatives}

The analysis is strictly limited to exI, prI, and inI in the context of 1 year of monitoring at $g=0.15$ followed by 4 non-monitored years, which was the scope of the original question posed to us. Alternatives such as different numbers of monitored and non-monitored years, different $g_{s}$ values, other ways of accounting for incidentals are beyond the scope of the study.

\section{References}

Dalthorp, Daniel, and Huso, Manuela, 2015, A framework for decision points to trigger adaptive management actions in long-term incidental take permits: U.S. Geological Survey Open-File Report 2015-1227, 88 p., http://dx.doi.org/10.3133/ofr20151227.

Dalthorp, Daniel, Huso, Manuela, and Dail, David, 2017, Evidence of absence (v2.0) software user guide: U.S. Geological Survey Data Series 1055, 109 p., https://doi.org/10.3133/ds1055. 
Publishing support provided by the U.S. Geological Survey Science Publishing Network, Tacoma Publishing Service Center

For more information concerning the research in this report, contact the Director, Western Fisheries Research Center

U.S. Geological Survey 6505 NE 65th Street

Seattle, Washington 98115-5016

https://www.usgs.gov/centers/wfrc 


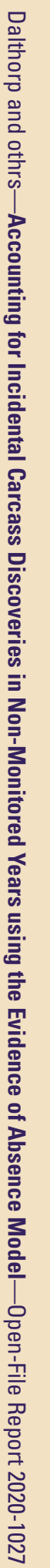

